\title{
A New Role for Telomerase in Promoting Meiotic Homolog Pairing Fidelity
}

Dana L. Smith ${ }^{1}$, Ashwini Oke ${ }^{2}$, Michael Pollard ${ }^{2}$, Carol M. Anderson ${ }^{2}$, Tangna Zhuge ${ }^{2}$, Phoebe Yam², Tatiana Gromova ${ }^{2}$, Kaylynn Conant ${ }^{2}$, Daniel B. Chu ${ }^{4}$, Neem J. Patel ${ }^{5}$, Fernanda Gonzalez ${ }^{2}$, Caitlin Stoddard ${ }^{1}$, Sean Burgess ${ }^{4}$, Andreas Hochwagen ${ }^{5}$, Wallace F. Marshall ${ }^{1}$, Elizabeth Blackburn ${ }^{1}$ and Jennifer C. Fung ${ }^{2 \#}$

${ }^{1}$ Dept. of Biochemistry and Biophysics, University of California, San Francisco, CA USA. ${ }^{2}$ Dept. of Obstetrics, Gynecology, and Reproductive Sciences and Center for Reproductive Sciences, University of California, San Francisco, CA USA.

${ }^{3}$ Dept. of Molecular and Cellular Biology, University of California, Davis, CA USA.

${ }^{4}$ Dept. of Biology, New York University, New York, NY USA.

${ }^{\#}$ Corresponding author.

\# Lead contact: tel +(1) 415 514-4309

e-mail: jennifer.fung@ucsf.edu

Key words: Telomerase, homolog pairing, SUN/KASH, meiosis, recombination, telomere, cytoskeleton, crossover, TLC1, infertility

Running title: Role of telomerase-mediated attachment in homolog pairing fidelity 


\begin{abstract}
Cytoskeletal forces acting upon telomeres promote active chromosome motion needed to pair homologous chromosomes during meiosis. The necessary components that allow this force to be applied to telomeres is still unclear, as are the roles of this motion and whether motion is needed primarily for increasing collisions of homologous regions, testing homolog pairing fidelity, or some other role. Here, we show a novel role for telomerase, previously known to be responsible for telomeric end replication, in anchoring telomeres to the nuclear envelope (NE) to provide proper transmission of cytoskeletal forces during meiosis. Reduction in telomerase function in Saccharomyces cerevisiae results in a dramatic decrease in the frequency of high velocity "pulls" resulting in earlier homolog synapsis and increased recombination. These observations are consistent with a model in which telomeric cytoskeletal engagement ensures homolog pairing fidelity by pulling apart improperly associated regions whereas general chromosomal motion aids in increasing homologous contacts.
\end{abstract}




\section{Introduction}

Segregation of homologous chromosomes is a key step in meiosis in which diploid cells halve their genome to produce haploid gametes. In humans, a leading cause of infertility, miscarriages and developmental disabilities is the failure to correctly segregate homologous chromosomes, resulting in aneuploidy (Hassold and Hunt, 2001). An early event in meiosis is the correct pairing of homologs. Given the large number of homologous sequence tracts found interspersed genome-wide among non-cognate chromosomes, an intriguing question is how homologs reliably find their correct partners (Zickler and Kleckner, 2015).

Many organisms show some degree of premeiotic homolog associations that are disrupted through unknown mechanisms prior to true meiotic pairing (Zickler, 2006). This phase is followed by the accumulation of chromosomal associations by recombinationindependent means (Church and Moens, 1976; MacQueen et al., 2005; Scherthan et al., 1996; Tsubouchi and Roeder, 2005). Once homologs are brought together in close proximity, homology assessment ensues. In most organisms, homology assessment is recombination dependent (C. elegans and Drosophila males being notable exceptions). Recombination initiates with Spo11-induced breaks (Keeney et al., 1997) that are resected to create 3' single-stranded DNA tails (Sun et al., 1991) These DNA tails invade the homolog to assess homology while also initiating DNA repair to form noncrossovers (NCOs) or crossovers (COs) that eventually form chiasmata.

Telomeres play an important role in chromosome reorganization via their attachment to the NE during meiosis. Telomeres cluster in proximity to the centrosome during meiotic leptotene, forming the telomere bouquet (Scherthan 2007). Although the bouquet disperses after zygotene, telomere-mediated connections to the SUN/KASH domain proteins in the NE remain throughout meiosis, connecting chromosomes with the cytoskeleton to promote chromosome motion (Hiraoka and Dernburg, 2009). Initially observed in yeast (Ding et al. 1998, Scherthan et al., 2007), telomere-led chromosome motion has been reported in other species including plants, flies, worms and mammals (Christophorou et al., 2015; Lee et al., 2015; Sheehan and Pawlowski, 2009; Wynne et al., 2012). In budding yeast, chromosomes move $\sim 0.3 \mu \mathrm{m} / \mathrm{sec}$ but sporadically move faster (>1 $\mu \mathrm{m} / \mathrm{sec}$ ) (Conrad et al., 2008; Scherthan et al, 2007). This motion results from a combination of nuclear deformation and cytoskeletal engagement with the telomeres. Many possible roles for this motion have been proposed (Figure 1A). Motion was first assumed to expedite contact between homologous regions (Ding et al., 1998), but 
current models propose that these forces may act to limit incorrect associations as well (Sato et al. 2009; Chacon et al., 2016; Marshall and Fung, 2018). Experiments perturbing this motion predict distinct consequences for meiosis depending on its functional role(s) (Figure 1B).

A

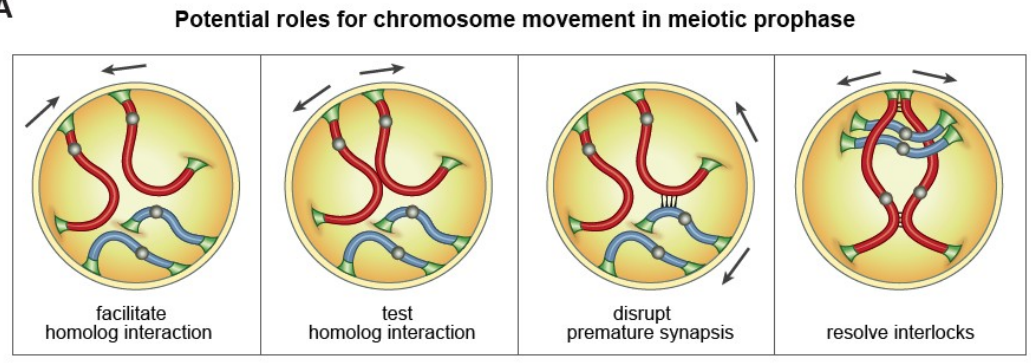

B

\begin{tabular}{|c|c|c|}
\hline $\begin{array}{l}\text { Model I: } \\
\text { Motion important } \\
\text { for pairing }\end{array}$ & & $\begin{array}{l}\text { Model II: } \\
\text { Motion important } \\
\text { for fidelity }\end{array}$ \\
\hline Meiotic measurement & Model I & Model II \\
\hline $\begin{array}{r}\text { gamete viability } \\
\text { pairing extent } \\
\text { time to pairing } \\
\text { synapsis extent } \\
\text { synapsis initiation } \\
\text { recombination }\end{array}$ & $\begin{array}{l}\text { poor } \\
\text { poor } \\
\text { delayed } \\
\text { incomplete } \\
\text { delayed } \\
\text { reduced }\end{array}$ & $\begin{array}{l}\text { moderately reduced } \\
\text { normal } \\
\text { normal } \\
\text { normal } \\
\text { faster } \\
\text { normal/increased }\end{array}$ \\
\hline
\end{tabular}

Figure 1: Models for role of active motion in meiosis.

A) Four models for the role of telomere-led chromosome movement: 1) motion brings homologs in close proximity; 2) motion pulls chromosomes apart to test homolog fidelity; 3 ) motion disassociates inappropriate synapsis and 4) motion removes interlocks or entanglements. B)

Different simple expectations for models I vs. II upon perturbation of motion.

To dissect the role of prophase chromosome motion, we disrupted the attachment of telomeres to the NE. In mitotic yeast cells, telomeres are tethered to the NE via two complexes (Schober et al., 2009) (Figure 2A). One complex tethers the subtelomeric chromatin and is made up of Sir4, a chromatin silencing protein, and Esc1, a protein found exclusively at the inner face of the NE (Taddei et al. 2004). A second complex includes components of the telomerase holoenzyme (Figure 2A) and anchors telomere distal ends to the NE. The telomerase-dependent attachment relies on the interactions of yKu with TLC1, the RNA component of telomerase, and Est1, a core protein component of telomerase, with the Mps3 SUN domain protein. (Schober et al. 2009). The functional importance of these two complexes in meiosis is not known.

In this study, we find that only the telomerase-dependent attachment is important for meiosis and gamete viability. Specifically, perturbation of telomerase results in fewer and less effective engagements of the telomere with the cytoskeleton, reducing the number of "pulls" a chromosome experiences as well as moderately decreasing the strength of the pulls. Our observations suggest these pulls are not needed to bring homologous 
chromosomes together but rather to test the fidelity of homologous associations by pulling apart incorrect associations.

A

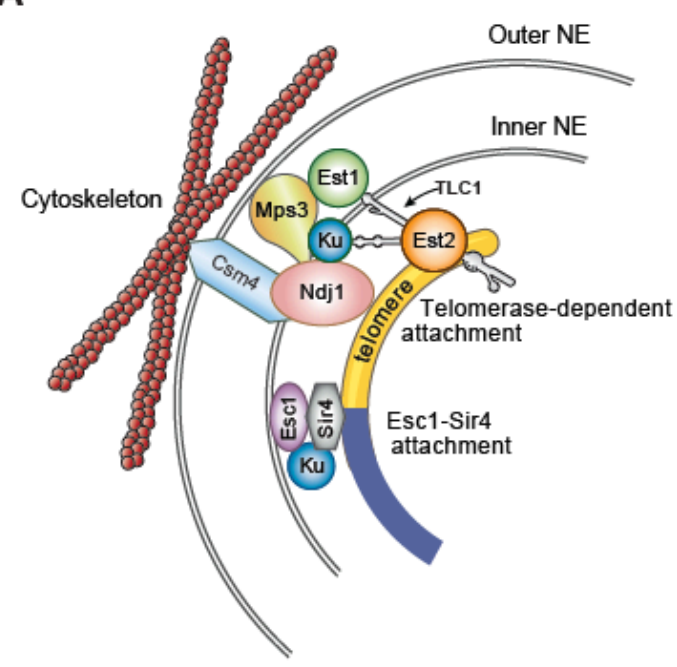

B

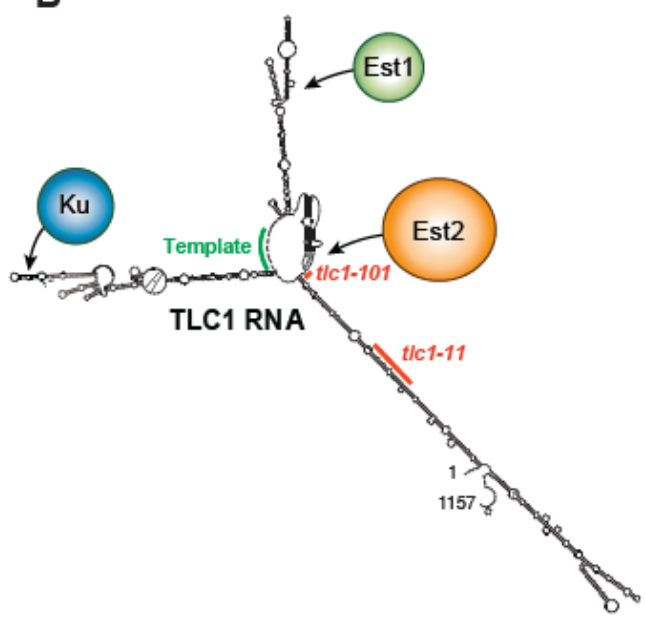

C

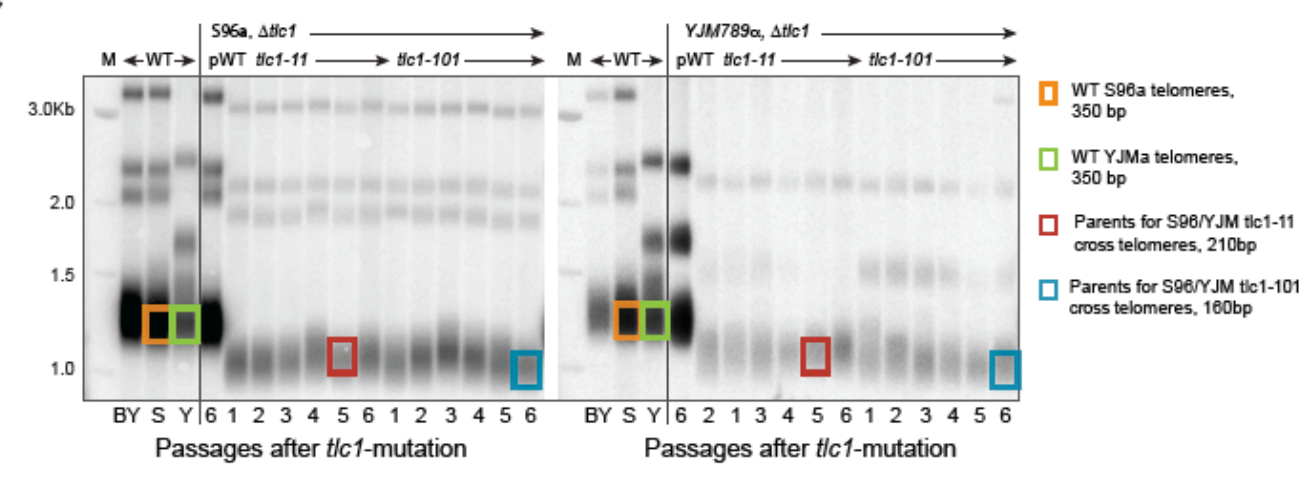

D

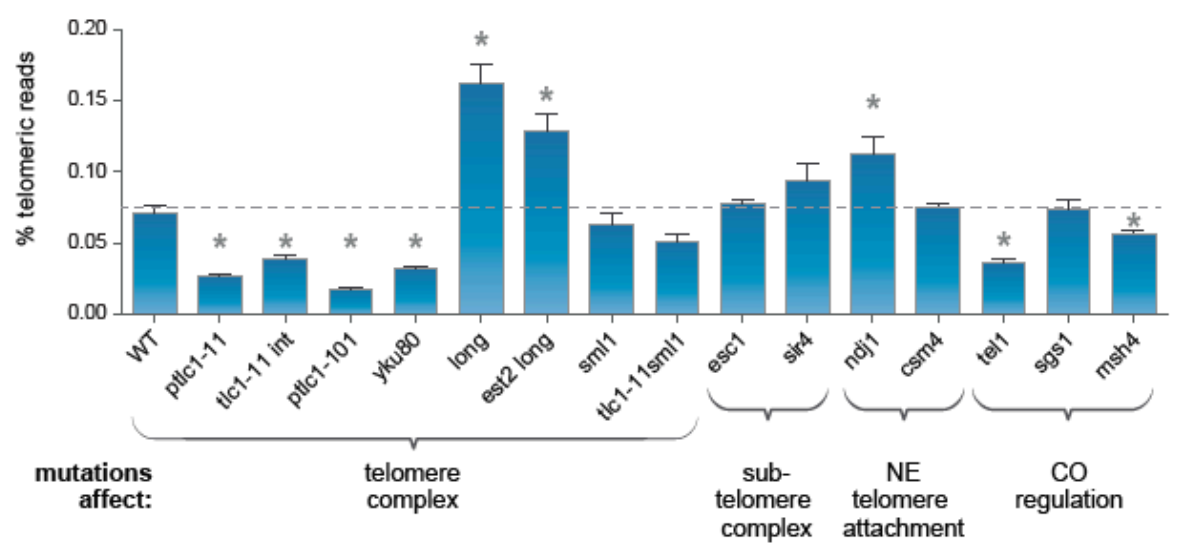

Figure 2. Two types of telomere attachments

A) Current model for mitotic telomere anchors to the NE. There are two types of telomere attachment to the NE: subtelomere and telomerase-dependent. Esc1 and Sir4 facilitate attachment of the subtelomere whereas the telomeric tip is attached through components of the telomerase holoenzyme. TLC1, the RNA portion of telomerase, binds both Ku and Est1; Est1 
binds Mps3, a protein firmly anchored in the NE. Ndj1 acts at the telomerase-dependent attachment during meiosis. B) Model showing the secondary structure of the TLC1 RNA with binding sites for Ku, Est1 and Est2. (Red) t/c1-101 and t/c1-11 mutations. C) Southern blot analysis of terminal Xhol restriction fragments to calculate telomere repeat size Generation of mutant alleles of TLC1. Cells were serially passed 6 times ( 120 generations). S96a and YJM789 t/c1 mutants with similarly short telomeres were chosen for mating and subsequent sequencing of gametes. DNA was probed with an $\gamma 32$ P-labeled 5'-(TGTGGG)4-3' Y'-specific probe. The lowest species represents the DNA fragment containing the terminal telomeric repeats. WT telomeres are about 350 bps; $t / c 1-11$ and $t / c 1-101$ are 210 and 160 bps respectively (For long telomere mutants see Figure S1). D) Relative telomere lengths determined from whole genome sequencing of WT and mutant spores in S96/YJM789. Yeast telomeres reads consist of a degenerate repeating sequence, $T$ followed by 1-3 Gs, TG-rich and AC-rich (complementary strand). Non-telomeric reads with long single letter runs arising from sequencing artifacts were removed.

\section{RESULTS}

\section{Mutants that affect telomere interaction with the NE}

To better understand the role of telomere NE attachment and motion in meiosis, we mutated the two complexes known to anchor telomeres to the yeast NE in mitotic cells: a sub-telomere-binding complex containing Sir4 and Esc1, and the distal telomeraseassociated complex containing core ribonucleoprotein components TLC1 and Est2 as well as the telomerase recruitment protein, yKu (Figure 2A, Table S1).

Deletion of SIR4 or ESC1 still allows long-term viability, whereas deleting telomerase holoenzyme genes causes telomeres to progressively shorten resulting in cell senescence. To study the effect on meiosis, we used two previously-characterized hypomorphic mutations in TLC1 that reduces levels of assembled Est2-TLC1 complex while partially reducing telomerase function (Lin et al., 2004; Figure 2B, map adapted from Hass and Zapulla 2015, and Figure S1). t/c1-101 is a 3-nucleotide substitution of bases $815-818$ in a region critical for the binding of TLC1 RNA to Est2p, the enzymatic protein portion of telomerase. t/c1-11 is a 30-bp deletion of nucleotides $850-880$ located in a region of uncharacterized function. $t / c 1-11$ and $t / c 1-101$ are viable long-term and maintain stable but shorter telomeres, an average of 210 and 160 bp respectively, compared to the WT length of about $350 \mathrm{bp}$ (Figure 2C).

To test telomerase function in meiosis per se without complications due to the telomere shortening, we created strains with pre-elongated telomeres by using the 
Cdc13-Est2 fusion protein, which lengthens telomeres by constitutively tethering telomerase to telomere ends (Evans and Lundblad, 1999). We lengthened telomeres to five times their normal length, before removing the CDC13-EST2-expressing plasmid, thus returning to normal telomerase recruitment. Using this technique, we examined strains with long telomeres, either with endogenous telomerase (EST2) "long" or without (est2L) "est2 long" (Figure S1B).

For comparison, we constructed additional mutant strains affecting telomere anchoring to the NE: $y k u 80 \Delta, n d j 1 \Delta$ and $c s m 4 \Delta$. Ndj1 is a meiosis-specific tether (Conrad et al., 1997) required for telomere attachment (Trelles-Sticken et al., 2000). Csm4 is a protein mediating the attachment to the cytoskeleton and is important for telomere clustering (Conrad et al. 2008; Kosaka et al., 2008; Wanat et al. 2008). We used whole genome sequencing to verify the relative lengths and sequence patterns of telomeres in our strains, by identifying telomeric reads that exhibit at least a $95 \%$ threshold for TG composition (Figure 2D; see Methods). This assay confirmed previously reported short telomere mutants (te/1 $\Delta, t / c 1-11, t / c 1-101)$, normal length telomere mutants (sir4 $\Delta$ and esc1 $1 \Delta$ ) and longer telomere lengths in the long mutants described above (Figure S1B). ndj1 $\Delta$ also exhibited longer telomeres in line with Ndj1's role in resetting telomere size by telomere rapid deletion (Joseph et al., 2005).

A

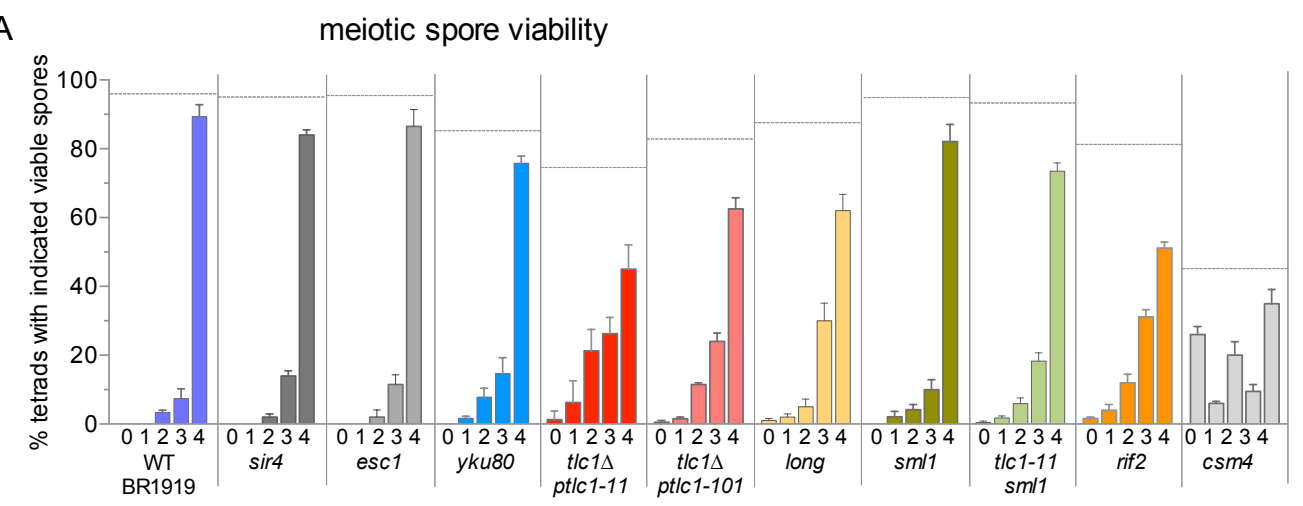

B meiotic progression
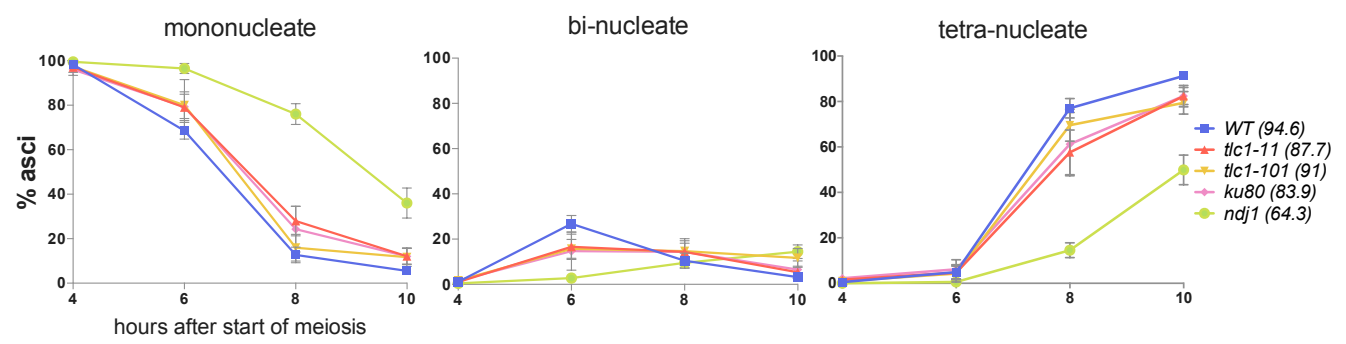
Figure 3. Analysis of spore viability and meiotic progression for t/c1-11

A) Spore viability measured in telomeric binding protein mutants in BR1919-8B. Spore viability, \% asci with $0,1,2,3$ or 4 viable spores. Dotted line indicates total spore viability. (See Figure S2 and Table S1 for other strains and mutants $(\mathrm{n}>200)$. B) Meiotic progression of t/c1-11, t/c1-101 and yku80 $\Delta$. Frequency of nuclear division measured by DAPI in SK1 ( $>3 \times 100 /$ timepoint).

\section{Disruption of telomerase-associated attachment results in reduced gamete viability and a slight delay in meiotic progression}

Although t/c1 strains have normal mitotic cell growth (Lin et al., 2004), in meiosis, spore viability is reduced (Figure 3A, Table S2). The mutants also exhibit a slight delay in meiotic progression (Figure 3B) and a slight reduction in overall sporulation frequency (Figure $3 A)$. In contrast, sir4 $\Delta$ and esc1 $\Delta$ have no effect on spore viability in the homozygous BR1919-8B (Figure 3A) or hybrid S96/YJM789 strains (Figure S2). tlc1-11, t/c1-101 and ku80 all enter meiosis I slightly later than WT (Figure 3B), but earlier than ndj1 1 (Conrad et al., 1997; Wu and Burgess, 2006). These findings suggest that optimal meiosis specifically requires the telomerase attachment for chromosome anchoring to the NE (together with meiosis-specific Ndj1), but not the subtelomeric Sir4-Esc1 attachment.

\section{Telomerase mutations change the frequency and velocity of chromosome "pulls"}

To determine whether meiotic chromosome motion is disrupted, we imaged single synapsed chromosomes in t/c-11 using Zip1-GFP (Methods). Zip1 is the synaptonemal complex (SC) protein responsible for synapsing paired homologs along their lengths (Sym and Roeder, 1993). In WT, the sixteen synapsing chromosomes make it impossible to definitively discern a single chromosome. Thus, we imaged chromosome motion in a zip3 $\Delta$ mutant, which stochastically initiates synapsis so that occasionally only one synapsed chromosome is observed, allowing for clear analysis of chromosome motion. Synapsis extent does not seem to be affected by zip3 $\Delta$ since the length of synapsis for the longest seen chromosome in zip3 $\Delta$ is comparable to WT (zip3 $\Delta 2.2+/$ $0.3 \mu \mathrm{m}$ vs. WT $2.2 \mu \mathrm{m}+/-0.3, \mathrm{P}=0.97)$. Motion in a t/c1-11 zip3 $\Delta \mathrm{BR} 1919-8 \mathrm{~B}$ strain containing Zip1-GFP was compared to zip3 $\Delta$ (Figure 4A, movies Figure S3A). Each 3D chromosome contour was traced using a semi-automatic method (Figure 4B). Figure 4C shows that a chromosome can traverse throughout the nucleus and is not confined to a limited region of the nucleus. The cumulative distance that telomeric regions (red and blue) travel is much greater than the cumulative distance of the midpoint (green), which 
occupies the center of the nucleus (Figure 4D). Moreover, each end of the chromosome moves independently of the other and motion is reduced further away from the telomere ends (Figure 4D), suggesting that force is not well transmitted along the full length of the chromosome. Consistent with earlier studies of meiotic chromosome motion (Scherthan et al, 2007; Conrad et al. 2008; Koszul et al., 2008), we found that chromosome motion consists of periods of relative quiescence ("dwells", shown in blue) alternating with sharp changes in velocity ("pulls", shown in red) (Figure 4E). In Figure 4F, a finer examination of the velocity profile is depicted. A pull is defined as any peak in the velocity vs. time graph greater than $10 \%$ of the maximum velocity, which allows us to determine both the magnitude and frequency of pulls. The "pulls" result from the chromosome end interacting with the cytoskeleton surrounding the NE (Scherthan et al., 2007). As summarized in Figure 4G, both the frequency and maximum velocity of the pulls are reduced in $t / c 1-11$, indicating that the mutation affecting telomerase-dependent attachment does perturb motion.

\section{Telomerase mutation does not alter telomere end declustering.}

In budding yeast, telomeres cluster in leptotene, to form the meiotic bouquet, which then disperses after zygotene. In Figure 4G, the cumulative distance traveled for telomere ends in $t / c 1-11$ is less than for WT, which not only indicates motion impairment but also raises the question whether telomeric ends can decluster normally. Immunofluorescene of Rap1 is commonly used to mark chromosome ends and to determine the level of telomere clustering. Trelles-Sticken et al. (2005) used Rap1 aggregation to reveal a problem of telomere bouquet declustering in the rec $8 \Delta$ mutant. However, our analysis reveals Rap1 aggregation in several telomere mutants (Figure $4 \mathrm{H}$ ) primarily reflecting differences in telomere length (Figure S3B-C), suggesting that the presence of Rap1 aggregates is not necessarily a reflection of telomere clustering. To directly assay telomere declustering, we asked whether two clustered $(<0.3 \mu \mathrm{m})$ chromosome ends in live cells in a zip3 vs. zip3 t/c1-11 could disperse. In WT ( $n=38)$ and tlc1-11 $(n=24)$ nuclei, all chromosomes ends that were initially clustered could disperse at least $>1 \mu \mathrm{m}$ apart indicating that declustering was not impaired in the mutant. 
A. Projection of SC

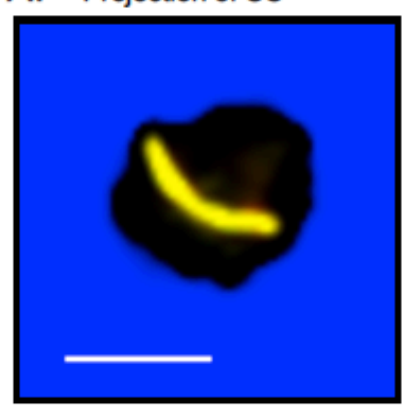

D.
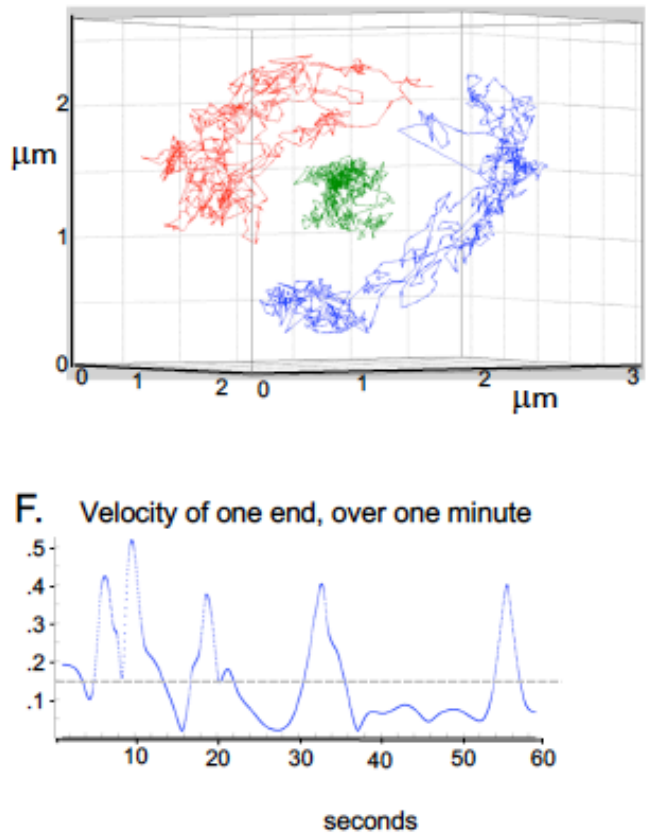

$\mathrm{H}$.
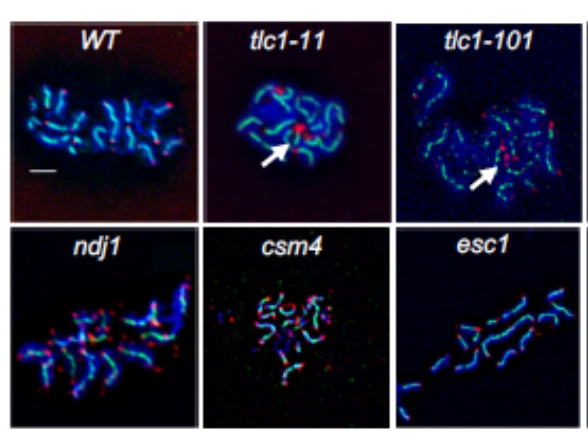

B. Digitization of SC

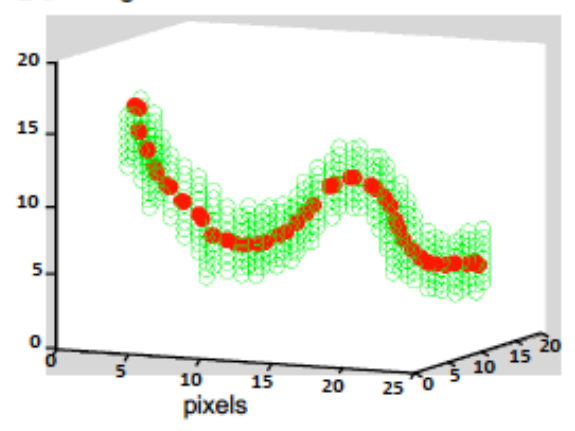

C. SC position through time

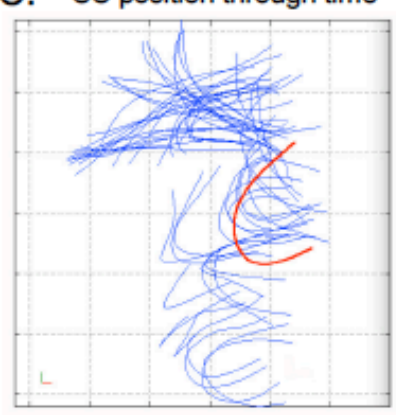

$\mu \mathrm{m}$

E. Velocity heat map, one end
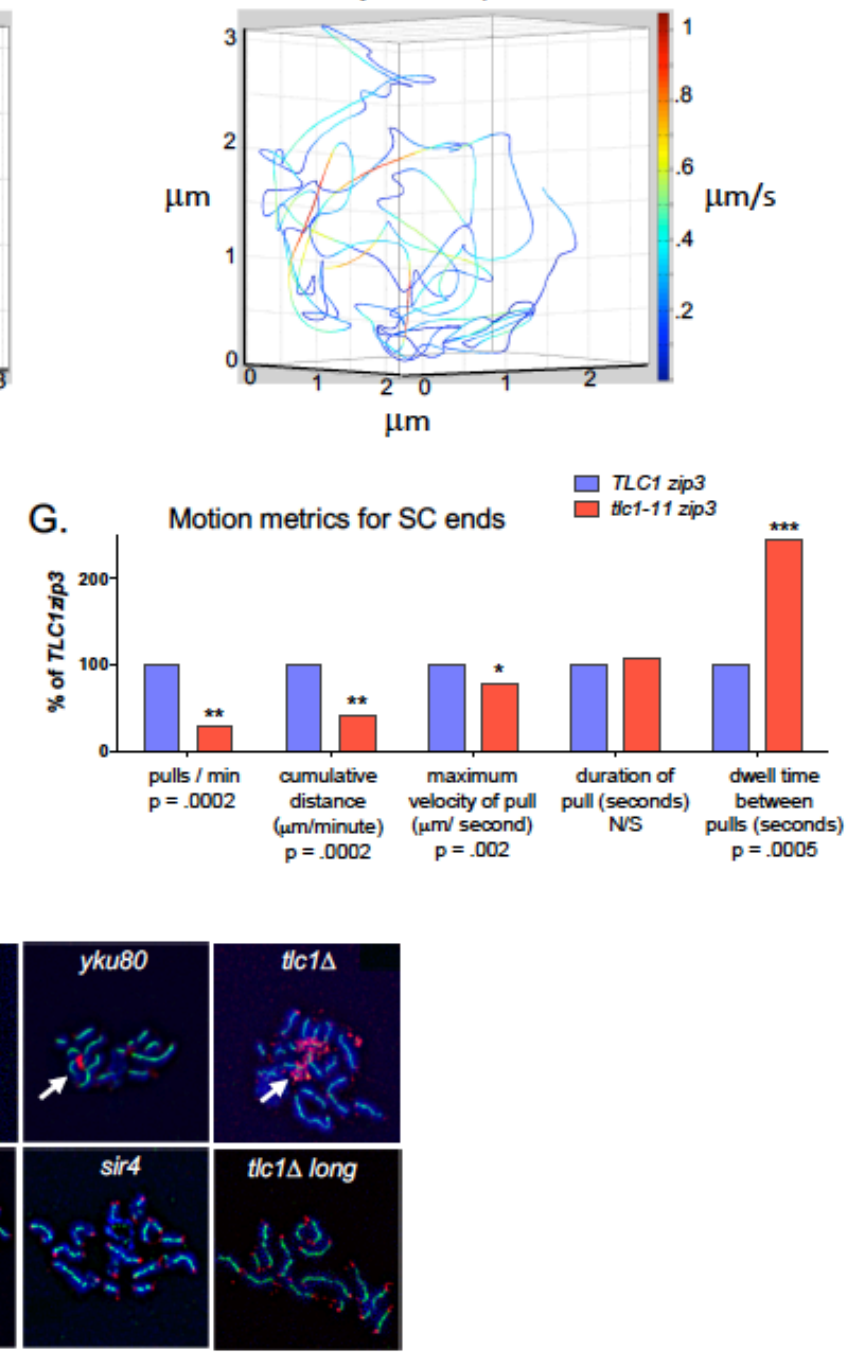

Figure 4. Analysis of telomere-led chromosome motion

A) Projection of a chromosome from a BR1919-8B zip3 meiotic nucleus containing a Zip1-GFP fusion highlighting extent of synapsis for a single chromosome (Scale bar, $2 \mu \mathrm{m}$ ). B) Digitized contour of the SC at one time point in 3D. Green circles represent segmented Zip1 intensity used 
to trace axial path of the SC through space (red circles) fitted to a quintic spline. C) Digitized contours throughout one time course. Red highlights a single contour. D) Ends move more than center of chromosome. Example from a t/c1-11 zip3 strain. Chain of displacement vectors plotted for each end of a chromosome (red and blue) and for the mid-point (green). E) Velocity heat map. Complete path traversed by the one telomeric end depicted in red in $\mathrm{F}$, in which color is proportional to velocity in a six minute timecourse. F) Changes of velocity of one end in one minute. Velocity of one end shown in E calculated from spline fit to particle path. Pulls are defined as periods of high velocity motion $>10 \%$ max velocity. Dwells are the periods between pulls. G) Changes of velocity magnitude and frequency in t/c1-11. Comparison of normalized SC motion metrics in t/c1-11 zip3 ( $n=53$ pulls over 29 minutes from 11 cells) or zip3 ( $n=154$ pulls over 24 minutes from 4 cells). * significant $\mathrm{P}$-values (t-test). H) Immunostained spread pachytene chromosomes. DAPI (blue) DNA, Zip1-GFP (Green) and Rap1 (Red). White arrows indicate representative telomeric aggregates of Rap1.

\section{Telomerase mutation perturbs engagement of chromosomal termini with the cytoskeleton}

We tested if the reduced frequency of telomere pulls was a consequence of perturbed telomere attachment to the NE and/or to the cytoskeleton. If t/c1-11 telomerase showed NE detachment, as reported for $n d j 1 \Delta$ (Trelles-Sticken et al. 2000), telomeres should localize towards the center of the nucleus rather than at the periphery.

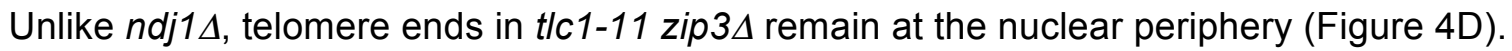
Therefore, we tested whether t/c1-11 affects the ability to maintain attachment to the cytoskeleton. Earlier detachment would result in shorter pull durations, while less efficient initial attachment events would cause less frequent (longer dwell times) but normal pull durations. Pull durations are the same with or without the t/c1-11 (Figure $4 G$ ), suggesting that $t / c 1-11$ is able to maintain attachment to the cytoskeleton once engaged. However, the reduction of average maximal velocity to $75 \%$ of WT (Figure $4 \mathrm{G}$ ) suggests that the attachment occurs but is not fully functional in t/c1-11. We also observed vastly longer dwell times between pulls (Figure 4G), indicating impaired ability to initiate pulls in this mutant. Hence t/c1-11 affects the frequency, or effectiveness, of initial engagement of the chromosome ends with the cytoskeleton 
A

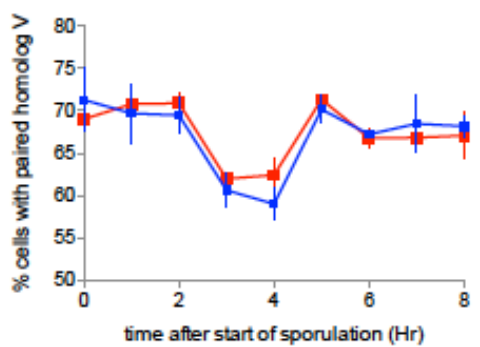

C TLC1+
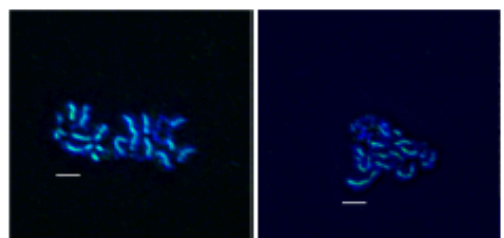

B

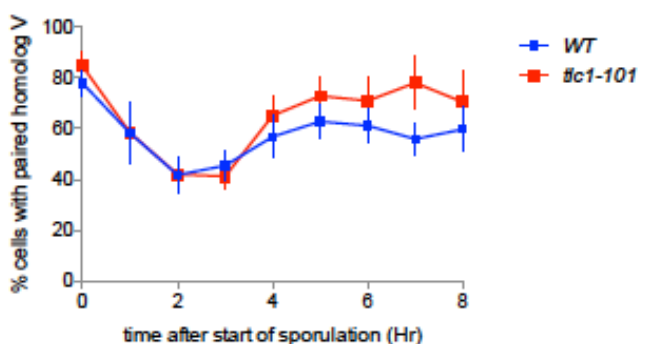

tlc1-11

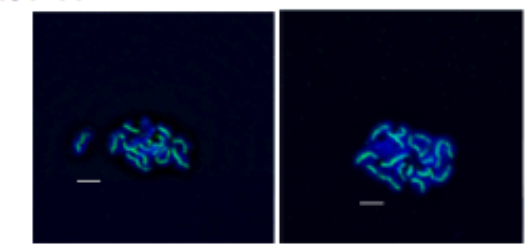

$\mathrm{D}$

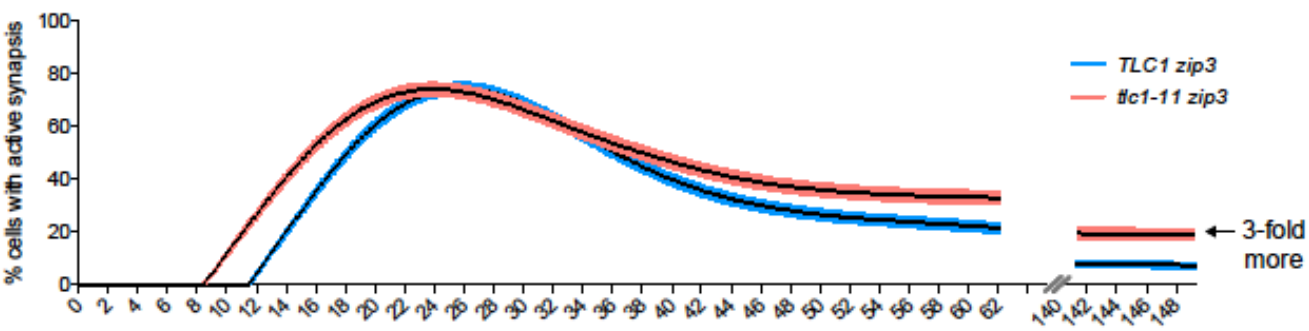

$\mathrm{E}$

hours after meiotic induction
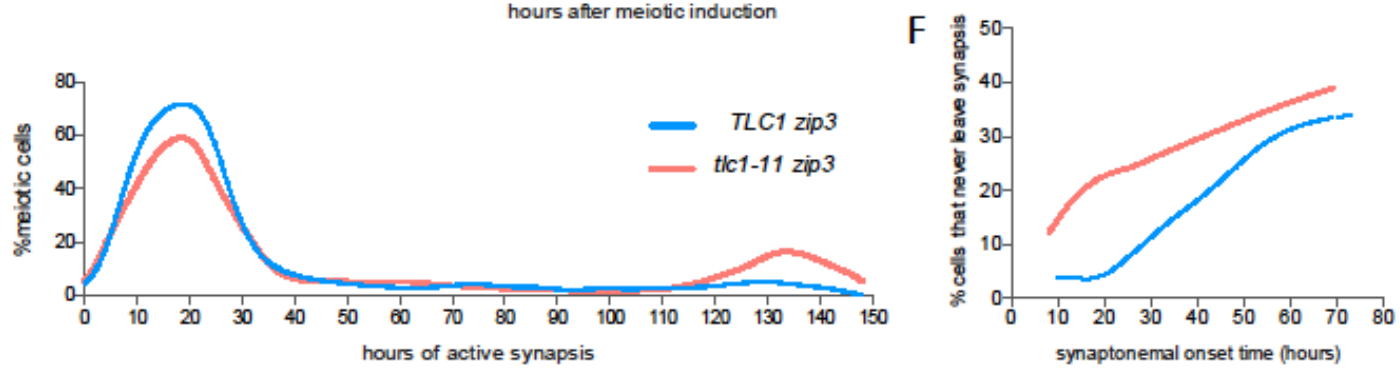

G

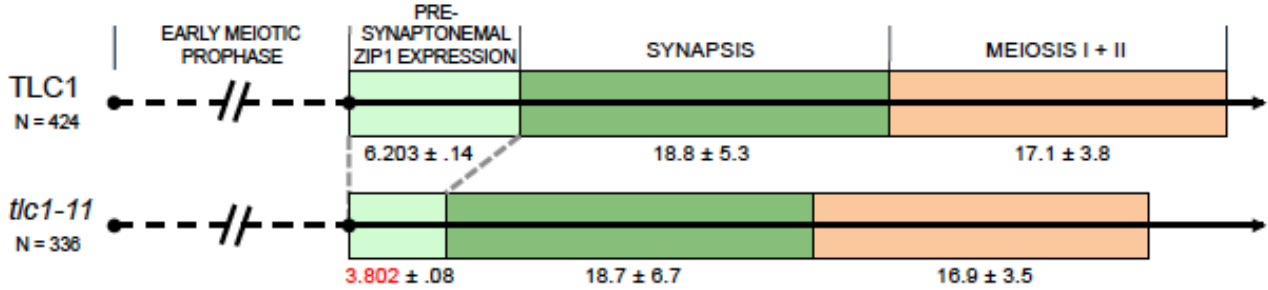

Figure 5. The t/c1-11 mutant exhibits early synapsis but normal pairing

A) Time and extent of pairing for t/c1-11. Cells with LacO repeats inserted near the centromere of each homolog were fixed hourly after meiotic start. LacO foci detected with Lacl-GFP are considered paired (1 spot) or unpaired ( 2 spots) $n=1000 /$ time point.

B) Time and extent of pairing for t/c1-101. Same as above. Scale bar $=2 \mu \mathrm{m}$. C) Extent of synapsis is normal in t/c111. Pachytene chromosomes were spread and immunostained against Zip1 for WT and tlc1-11. D) Synapsis initiates earlier in t/c1-11. Individual t/c1-11 zip3 $(n=336)$ or zip3 $(n=424)$ cells containing Zip1-GFP are tracked in vivo throughout the entire course of meiosis. Time to synapsis initiation was recorded. At the end of the live cell tracking a three-fold increase in cells were seen 
that still were in active synapsis. E) Analysis of synapsis duration. The time from synapsis initiation to synapsis termination was determined for zip3 and $t / c 1-11$ zip3. For $t / c 1-11, \sim 20 \%$ of the population showed extremely long synapsis durations. F) Cells showing long synapsis durations start synapsis throughout time course, not only at late times. Time from induction to synapsis onset are recorded for cells with long synapsis durations. G) Early synapsis is due to shorter presynapsis period. Summary of major periods of meiosis determined from single cell in vivo tracking until completion of meiosis.

\section{Homolog pairing kinetics are unaffected by telomerase dependent attachment site mutations.}

If chromosome pulls help homologs locate their partners more efficiently, disruption of telomerase-dependent attachments should lead to slower pairing kinetics.

Alternatively, if this chromosome motion is mainly used to test homolog interaction and disrupt pairing mistakes, initial pairing kinetics should be minimally affected by reduced telomere motion. To distinguish between these possibilities, we examined the timing and extent of homologous pairing by fluorescently marking a site near the centromere of chromosome $\mathrm{V}$ of each homolog with a 256-LacO array in cells also expressing GFPLacl repressor protein. We generated pairing time courses for WT, t/c1-11 and t/c1-101 in the highly synchronous SK1 strain. Both the t/c1-11 and t/c1-101 mutants progress with WT kinetics (Figure 5A and B). Pairing kinetics of a telomere proximal site in t/c1-11 and t/c1-101 are similar to WT (Figure S4A). The unperturbed timing and extent of homologous pairing we observe in the $t / c 1$ strains is not consistent with a primary role for chromosome motion to speed up pairing, suggesting the alternative model (Figure 1B).

\section{Telomerase mutation leads to precocious synapsis}

We next analyzed the timing of synapsis in t/c1-11 on the grounds that if chromosome motion aids pairing, then telomerase mutants should delay synapsis which cannot occur until pairing has completed. Alternatively, if chromosome pulling acts to pull incorrectly paired chromosomes apart, reduced motion should lead to more rapid completion of pairing, since the time spent undoing incorrect associations and researching for correct homologs would be eliminated. In pachytene chromosome spreads, t/c1 mutants achieve full WT synapsis (Figure $5 \mathrm{C}$ ). Although synapsis extent is not perturbed, live imaging of individual homolog pairs in single cells reveals that t/c1-11 zip3 $\Delta$ starts synapsis fully $2.5+/-0.11$ hours before zip3 $\Delta$ (Figure 5D). Despite initiating synapsis earlier, the absolute duration of synapsis for the majority of individual $t / c 1-11$ 
zip3 $\Delta$ cells is the same as in zip3 $\Delta$ (18.7 vs. 18.8 hours) (Figure $5 E$ ). Notably, $\sim 20 \%$ of t/c1-11 zip3 $\Delta$ cells remain indefinitely in the process of synapsis assembly and disassembly thus failing to exit meiosis I (Figure 5E). Figure 5F shows that the subset that fails to exit synapsis includes cells that initiate early as well as throughout the time course. The inability to finish synapsis in a small subset of the population is consistent with a subset of cells containing unsynapsed chromosomes that prohibit meiotic progression due to the synapsis checkpoint (reviewed in Macqueen and Hochwagen 2011). Corroborating evidence for a meiotic checkpoint was found in the meiotic progression assays (Figure 3B). Relative to WT, t/c1-11, t/c1-101 and $\Delta k u 80$ mutant strains showed the time course patterns expected if a small subpopulation of cells fails to progress past meiosis I. Consistent with Fig 1B model II, precocious synapsis is an expected outcome when motion is perturbed, and further suggests that chromosome dynamics plays an important role in testing homolog fidelity.

\section{Higher recombination results when motion is perturbed by mutation of telomerase dependent attachments.}

An additional function of synapsis is to promote interhomolog recombination. Since synapsis initiates earlier in telomerase mutants, we examined whether recombination was altered. To gain an accurate representation of COs and NCOs genome-wide, we measured recombination by whole genome sequencing (RecSeq) in the S96/YJM789 hybrid strain containing over 60,000 SNPs (Anderson et al., 2011). Mutants affecting the telomerase-dependent but not the sub-telomeric NE attachment led to a genome-wide increase in COs (Figure 6A), whether or not telomeres were made short or overelongated. NCOs also increased in proportion to COs (Figure 6B). In contrast, $\Delta t e l 1$ and $\Delta s g s 1$ mutations also increase COs but alter the ratio of COs to NCOs. Such ratio alterations indicate changes in $\mathrm{CO}$ regulation (Anderson et al., 2015) suggesting that this aspect of regulation is unaffected in telomerase mutants. Interestingly, esc1 $1 \Delta$ also results in an altered $\mathrm{CO} / \mathrm{NCO}$ ratio potentially suggesting an undiscovered role for Esc1 in recombination. Importantly, the recombination increase is not restricted to telomeric regions but occurs chromosome-wide (Figure 6C). Contrary to the expectation of altered recombination due to ectopic pairing, the distribution of recombination signatures is identical between t/c1-11 and WT indicating that the increased recombination for the most part is normal (Figure 6D). The lack of any observed recombination errors is likely due to the fact that most of the ectopic recombination is occurring in the $20 \%$ subset of 
nuclei that fail to exit meiosis (Figure 5E) which do not make 4-spore viable tetrads used in the RecSeq analysis. Finally, in all these mutants, overall interference decreases moderately (Figure 6E). Together with the observation of early synapsis, the high level of error-free recombination suggests that perturbing the pulling forces on the chromosome allows for the chromosomes to associate earlier permitting more time for interhomolog recombination to occur. This provides further evidence for a model in which motion is needed to dissociate chromosomes as a means to test homology whether they correctly or incorrectly paired.
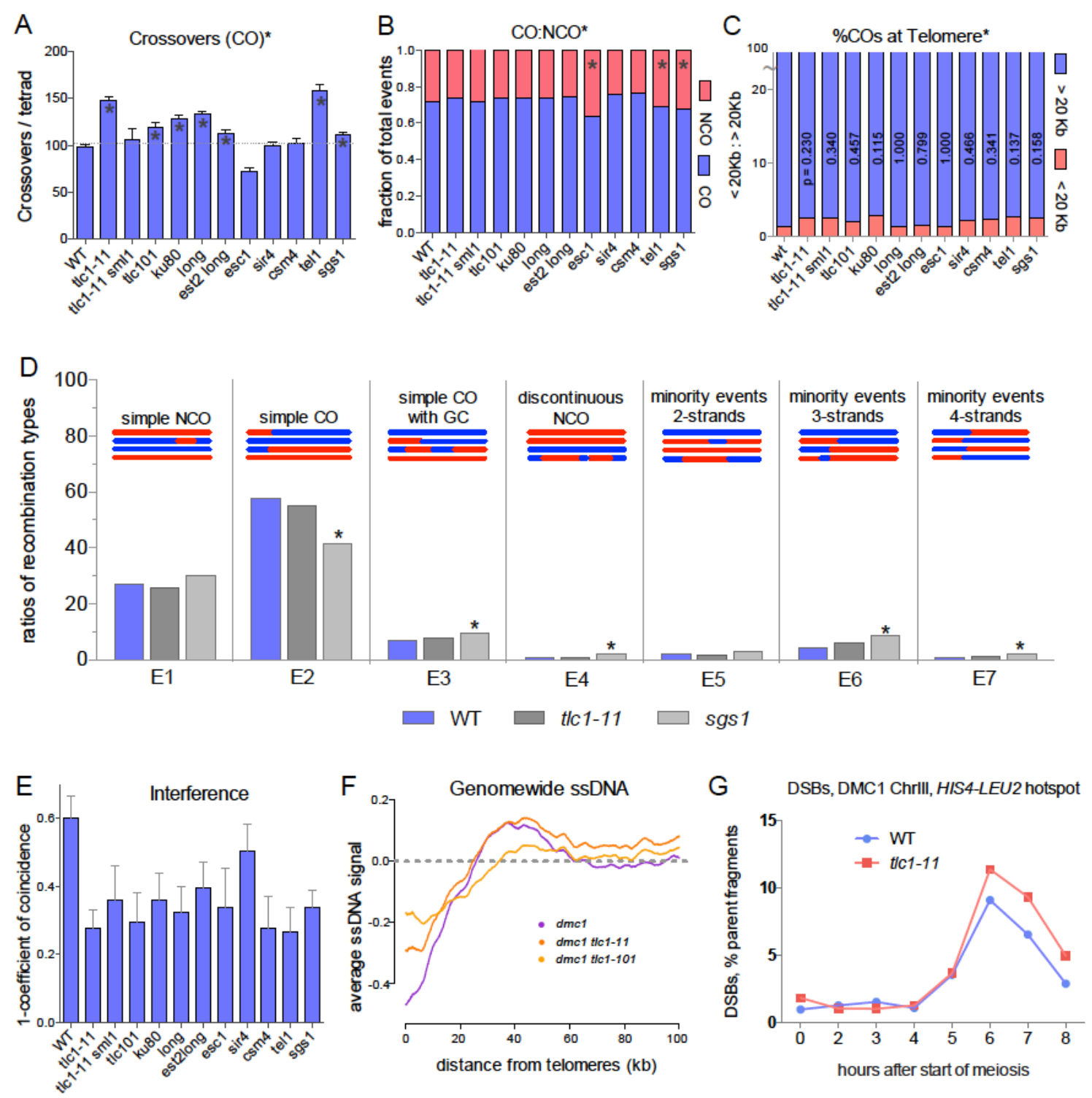

Figure 6 Higher recombination is observed for mutants with reduced telomerase 
A) More COs in telomerase deficient mutants. Average COs per meiosis obtained from sequencing analysis. * chromosome 12 is removed from all strains for comparison purposes due to YJM789 chromosome 12 disomy in some strains. B) Ratio of total COs vs.NCOs (CO:NCO). C) CO frequency at telomere proximal vs. distal. P-values are from chi-square analysis of $\mathrm{COs}<$ $20 \mathrm{~Kb}$ from the telomere end (red) and $\mathrm{COs}>20 \mathrm{~Kb}$ from the end (blue) for each mutant and WT. D) Comparison of recombination signatures between WT, t/c1-11 and sgs1. E) Average CO interference calculated from coincidence of adjacent intervals genome-wide. F) DSB levels measured using genome-wide ssDNA signal in dmc1 SK1 as a function of distance from telomere. G) Timing and frequency of DSBs as percentage of parent fragments at the HIS4-LEU2 DSB hotspot on chromosome III in a DMC1+ SK1 strain, (See Figure S6)

A CO increase can reflect either an increase in DSBs or a change of bias toward interhomolog repair of breaks. We measured DSBs by genome-wide detection of ssDNA in a $d m c 1 \Delta$ background that allows ssDNA regions to be stably maintained at the sites of DSBs. When the DSBs of chromosome VIII were measured in $d m c 1 \Delta$, neither $t / c 1-11$ $d m c 1 \Delta$ nor t/c1-101 dmc1 $\Delta$ showed more DSBs than $d m c 1 \Delta$ alone (Figure 6F, Figure $\mathrm{S} 5 \mathrm{~A}, \mathrm{~B})$. Although deletion of DMC1 facilitates the preservation and detection of DSBs, it can potentially mask DSBs introduced later in meiosis (Thacker et al. 2014). We therefore measured DSBs at a single break site, the HIS4-LEU2 hotspot on chromosome III without perturbing DSB processing (DMC1). Again, the number of DSBs in the t/c1 mutants is similar to WT (Figure 6G, Figure S5C and D). We conclude that the increased recombination is due to a change in bias toward interhomolog DSB repair, consistent with the earlier association of the homologs as revealed by the early synapsis (Figure 5D).

\section{Pairing simulations predict that lowering the frequency of cytoskeletal attachments will cause increased association of chromosomes.}

Although t/c1-11 alters telomere motion but does not eliminate it, it is unclear whether the quantitative reduction in the frequency or velocity could in principle be sufficient to alter chromosome association. We have previously used a computational model based on Brownian dynamics simulations (Marshall and Fung, 2016), to show that forces applied to telomeres can, in principle, promote pairing fidelity by pulling weakly paired regions apart (Marshall and Fung, 2018). By allowing chromosomes to associate reversibly (Figure 7A), with the affinity of two chromosomes described by the rate at which paired loci become unpaired, we can ask what happens if we reduce 
motion. In simulations in which chromosomes are allowed to either pair with their correct homolog or an incorrect homeologous chromosome, with different off-rates, it is frequently seen that chromosomes will pair with the wrong chromosome even when the off rate is different by an order of magnitude (Figure 7B). Simulations showed that stretches of incorrect pairing were eventually pulled apart (Figure 7C), whereas in the absence of telomere forces, incorrectly paired regions remained associated for long periods of time (Figure 7D). The apparently stable association of incorrect regions, despite the high unpairing rate of individual loci, is due to avidity effects. When simulating homolog pairing with the observed reduction in the frequency of high velocity pulls, we found that chromosomes misassociated $25 \%$ more frequently. Because our measurements showed not only a change in frequency but also a change in velocity, we also simulated homolog pairing with normal frequency of pulls but with a $75 \%$ reduction in velocity. This was also sufficient to increase the rate of mispairing. Reduction in either the frequency or velocity of the telomere pulling motions to an extent matching our experimental observations led to an increase in incorrect chromosome associations. Combining a $25 \%$ reduction in frequency with a $75 \%$ reduction in velocity, which mimics our experimental observations of the t/c1 mutant in living yeast cells, led to a more dramatic effect (Figure 7E). We conclude that, based on our computational model, a quantitative reduction in either the frequency or velocity of telomere pulls, such as we have observed in t/c1 mutants, could be enough to increase mispairing rates.

\section{DISCUSSION}

\section{Telomerase-dependent attachments modulate cytoskeletal forces during meiosis}

In most organisms, the cytoskeleton is attached to chromosome ends through the nuclear membrane via the SUN/KASH domain proteins that connect to proteins that bind to the telomere. In budding yeast, although it was long known that Ndj1 is important for tethering the telomere to the nuclear membrane in meiosis, there has been a question of whether other telomeric components are required. Recently in mouse, MAJIN (Membrane-Anchored Junction Protein) was found to link telomeric DNA to the NE by exchanging the telomere cap (Shibuya et al, 2015). In our study, we show that telomerase is important for full conduction of cytoskeletal forces during prophase I revealing an important mechanical role for telomerase in meiosis. We see that reduced telomerase function in t/c1-11 alters both the velocity and frequency of telomere-led 
motions, resulting in earlier initiation of synapsis, a subpopulation of cells that are unable to exit synapsis, an increase of crossing over, and lower spore viability. In contrast, mutants of the Esc1-Sir4 subtelomeric attachment show no reduction in spore viability or increase in overall recombination, suggesting that this anchoring mechanism is not critical for meiosis. One reason the Esc1-Sir4 attachment might be superfluous in meiosis is the presence of $\mathrm{Ndj} 1$. Ndj1 may provide greater strength to the telomerase dependent attachment in order to withstand the cytoskeletal forces experienced in meiosis but not in mitosis.

A

B

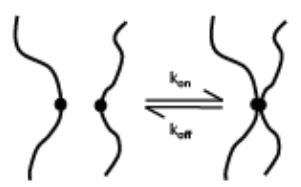

Pairing: 2 simulations for 2 pairs of chromosomes, with partners

C

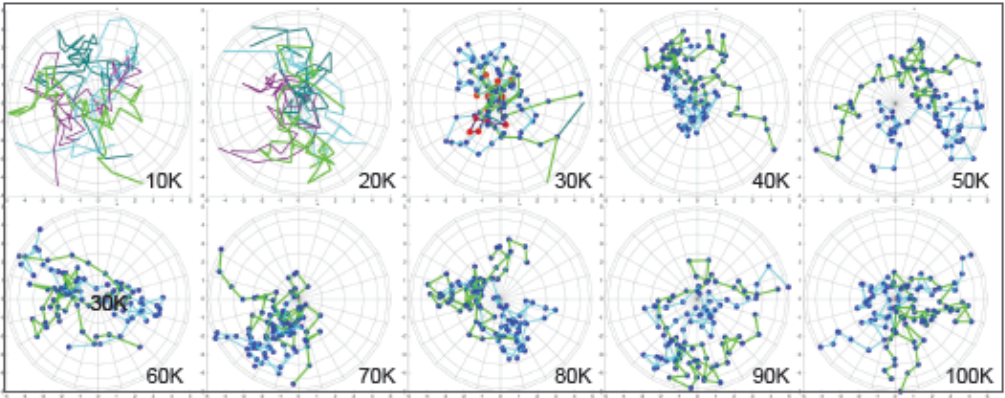

D

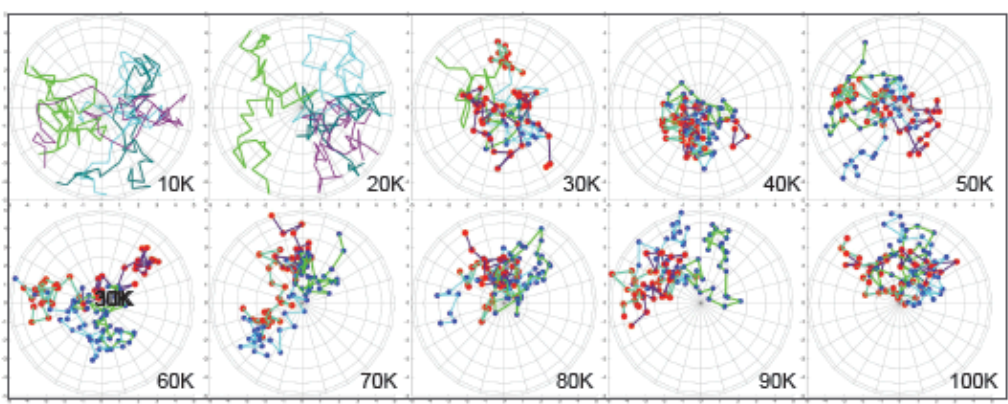

E

F
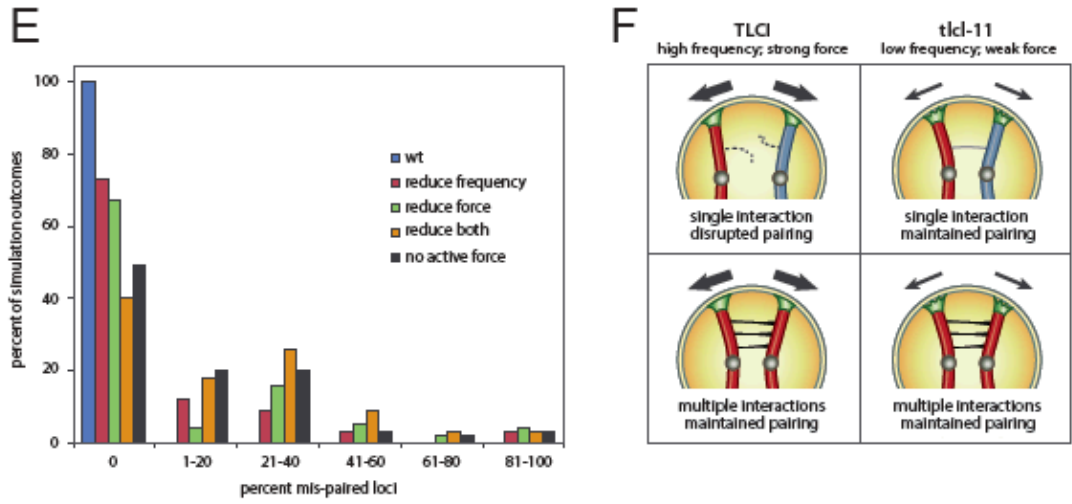
Figure 7 Simulation of active random forces predicts pairing errors from reduced pulling frequency.

A) Pairing as a reversible process. Individual loci can pair when they randomly move within some capture radius. The probability of such a pairing event depends on the random motion of the chromosomes (Marshall and Fung, 2016). Once paired, any locus has a probability $\mathrm{p}_{\text {(unpair) }}$ of becoming unpaired at any given time in the simulation. Correctly paired loci would have a small value of $\mathrm{p}_{\text {(unpair) }}$ and incorrect loci a high value. B) Pairing diagrams for simulations with two pairs of chromosomes. Blue and purple lines represent one pair of homologs, while orange and red represent a second pair of homologs. Correct pairing is blue pairing to purple and orange pairing to red. Diagrams plot final results as four parallel lines, where each pair of lines represents one of the four chromosomes on the left along with a plot of its pairing partner on the right. The left panel depicts correct pairing for approximately $3 / 4$ the length of all four chromosomes, with mispairing at one end. The right panel shows multiple correct and incorrect pairings for all chromosomes. C) Example of simulation $r$ with $100 \%$ velocity and normal pulling frequency. Dark green and bright green represent one homolog pair and purple and blue represent a competing homolog pair. Red dot indicates a mis-paired locus and a blue dot indicates a correctly paired locus. In this WT simulation, both homolog pairs eventually become correctly associated. Examples taken at every 10,000 timepoints (10K) out of 100K. D) Example of one simulation run with $100 \%$ velocity but no pulling. Without any pulling forces, example runs will show incorrect association at the end of the run. E) Simulating five scenarios of the effect of alteration in magnitude and frequency of telomere-led active forces. WT denotes an active telomere force equal to four times the thermal random force and a pull frequency of 1 , meaning that the pulling force is constantly being exerted. "reduce frequency" keeps the high pulling force but reduces the pulling frequency to $25 \%$. "reduce force" keeps a pulling frequency of $100 \%$ but reduced the pulling force to $75 \%$ of its full value. "reduce both" means that pulling frequency is $25 \%$ and pulling force is $75 \%$ of maximal. "no active force" means that no additional force is exerted on the telomeres other than thermal forces. The graph plots the fraction, out of 100 independent simulation runs, in which the final paired state has a given fraction of loci incorrectly paired. The first bin contains only those outcomes in which pairing was perfect, i.e. no mistakes. All other bins contain the range of mis-pairing fractions indicated.

\section{Is the structural role for telomerase related to its role in end replication?}

How do telomerase mutations alter NE interactions? The effect could be direct, with telomerase interacting with NE proteins such as Mps3, as has been reported in mitosis (Schober et al., 2009). Alternatively, telomerase's role might be indirect, through its effect on end-replication. Since cohesin loading occurs during replication (UhImann and Nasmyth, 1998), impairment of telomerase function during replication might affect the 
loading of meiotic cohesin Rec8, leading to a loss of sister cohesion at the telomeres and subsequent weakening or altering the attachment site. In this scenario, chromosome movement could be disrupted if each sister chromatid interacts with different actin fibers, such that the two sisters are pulled in different directions, creating a stalemate in which the chromosome end does not move. Interestingly, both in yeast (Trelles-Sticken et al., 2005) and mouse (Revenkova et al., 2004), removal of Rec8 or the meiotic specific cohesin subunit SMC1 $\beta$, result in the inability to disperse telomere clusters. In the case of budding yeast, deletion of REC8 has been directly shown to affect chromosome velocity in meiosis (Trelles-Sticken et al., 2005). However, these mutants also affect cohesion along the arms. Another argument linking replication to attachment dysfunction is our observation that removal of Sml1 suppressed several t/c1-11 meiotic defects. Sml1 inhibits ribonucleotide reductase (RNR) needed in the rate-limiting step in dNTP production (Reichard, 1988). Deletion of Sml1 improves replication by suppressing replication fork stalling. Our finding that that $s m / 1 \Delta t / c 1-11$ improves spore viability (Figure $3 \mathrm{~A}$ ) and recombination (Figure 6A) over t/c1-11 alone in addition to reducing telomere shortening (Figure 2D), indicates improved telomere replication. Replication fork stalling at telomeres has previously been found to increase with loss of telomerase in mitotic cells, an increase that was also suppressed by Sml1 deletion (Xie et al., 2015; Jay et al., 2016). Thus, sm/1-dependent suppression of replication fork stalling restores telomerase-deficient telomere integrity, and potentially restores the ability to properly incorporate cohesins. Telomerase is clearly key to full transmission of cytoskeletal forces, but whether it plays a structural role or acts indirectly through ensuring sister chromatid cohesion will be an interesting subject for future studies.

\section{Active Motion Ensures Fidelity of Homologous Pairing}

The importance of chromosome motion for homolog pairing was first proposed after the discovery that active chromosome motion is driven by cytoskeletal forces during prophase I in S. pombe (Chikashige et al. 1994). Since then it has been further established that active motion speeds up the search for homologous sequences both experimentally (Conrad et al., 2008; Kosaka et al., 2008; Mine-Hattab and Rothstein, 2012) and theoretically (Marshall and Fung, 2016). While active motion may facilitate homologs locating each other within the nucleus, at least three lines of evidence argue that this is not the only essential role for telomere-led forces. First, although pairing is delayed in the $n d j 1 \Delta$ mutant, which abrogates tethering to the NE, the extent of pairing of 
individual loci eventually reaches near WT levels (Trelles-Stricken et al., 2000). Second, Chacon et al, 2016 show that transiently halting active chromosome motion in fission yeast results in excessively stable associations forming at loci that were already paired when the motion was halted, thus indicating a second role for the motion that acts after initial pairing. Third, our study shows that by particularly perturbing the chromosome pulls leads to earlier synapsis even though timing and extent of pairing occur normally, providing evidence that telomere-led motion normally acts to delay a step that occurs after initial pairing and before synapsis. We propose that this additional step is the removal of incorrect associations.

\section{Model for removal of incorrect associations without invoking a molecular sensor}

Sato et al (2009) proposed that active forces are used to sense tension between associated pairing centers. Their model uses tension to probe the correctness of association in order to generate a global signaling trigger for initiation of synapsis. An alternative model is that active forces simply act to pull apart incorrectly associated regions, but aren't strong enough to pull apart long stretches of correctly paired regions, such that active force favors the persistence of correct, but not incorrect, associations (Figure 7F). This simpler model does not posit any signals or tension sensors, but simply suggests that the active forces would reduce the extent of incorrect pairing relative to correct pairing. Computational simulations of this model indicate that random forces can provide a high level of selectivity in favoring correct over incorrect associations (Marshall and Fung, 2018).

\section{The increased recombination in t/c1-11 reflects early but not prolonged homologous engagement}

Chacon et al. (2016) reported that the temporary elimination of cytoskeletal forces stops pairing oscillations of paired ade 3 loci and, upon resumption of motion, resulted in an increase in ade3 missegregation, which was not observed in ade 3 loci that were not already paired at the moment the motion was stopped. This missegregation was rescued by overexpression of the $\mathrm{CO}$ resolvase Mus81 and was interpreted to mean that without oscillations, irresolvable recombination intermediates form. In contrast, in t/c1-11, pairing oscillations still occur but the stronger velocity pulls were less frequent and reduced in magnitude. Unlike the situation observed in Chacon et al. (2016), recombination is essentially normal in 4-spore viable t/c1-11 as seen by the WT CO/NCO ratio (Figure 
6B) and lacks any difference in recombination signatures (Figure 6D). Together these results suggest that perhaps there are two components to the motion that contributes to homolog fidelity; one that helps correct small misalignments (pairing oscillations) and one that can correct major misalignments (strong velocity pulls).

In summary, disrupting or reducing telomerase function reduces homolog-pairing fidelity, a conserved crucial aspect of successful meiosis. These findings may implicate a new direction for investigating the function of telomerase in ensuring fertility in humans.

\section{Methods}

\section{Strain Construction}

All yeast strains are derivatives of the SK1, BR1919-8B, S96/YJM789 hybrid or S288C backgrounds as detailed in Table S1. Complete disruption of ORFs was carried out by PCR-mediated gene disruption. For $t / c 1-101$ and $t / c 1-11$, both integrated and plasmid-containing strains were made. To integrate the t/c1 mutant alleles, loop-in, loopout of a mutation-containing plasmid was followed by PCR verification to replace TLC1. Alternatively, a plasmid containing the specific mutant allele was transformed into $t / c 1 \Delta$. For t/c1 alleles, haploid strains were streaked serially 6 times to ensure complete penetrance of mutant telomere phenotypes ( 2 days of growth on solid media at $30^{\circ} \mathrm{C}$ represents approximately 20 generations). To create diploid strains for subsequent sequencing, imaging and meiotic progression assays, a and $\alpha$ parents with telomeres of similar genotype and length were mated. For recombination mapping, S96/YJM789 hybrid diploids were sporulated. Spore viability tests were carried out in BR1919-8B and S96/YJM789. The BR1919-8B background was used for genetic recombination, chromosome spreads, synapsis measurement and movement assays. Synchronous meiotic progression assays (FACS analysis of replication onset, pairing, DSB formation and asci formation) were carried out in SK1. Strains are listed in Table S1. Detailed strain genotypes as well as plasmid and oligo sequences are available upon request.

\section{Southern Blotting Analysis of Telomere Length}

Genomic DNA was prepared from haploid cells from serial streaks on solid media after the indicated number of passages. Genomic DNA was then digested with Xhol and run on $0.8 \%$ agarose gels. DNA was transferred from the gels to Hybond $\mathrm{N}+$ membranes and probed with $\gamma 32 \mathrm{P}$ end-labeled WT telomeric repeat oligonucleotide (TGTGGTGTGTGGGTGTGGTGT) and visualized using a phosphoimager. 


\section{Meiotic Time Courses}

To induce synchronous meiosis, diploid strains were pre-inoculated at OD600 $=0.3$ in BYTA medium. Samples were collected and fixed in $70 \%$ ethanol for time and every $15 \mathrm{~min}$ for for 2 hours. Cells were analyzed by fluorescence-activated cell sorting (FACS). Ethanol-fixed cells were pelleted, washed twice in $50 \mathrm{mM}$ sodium citrate, $\mathrm{pH}$ 7.5 , and resuspended in $600 \mu \mathrm{l}$ of $50 \mathrm{mM}$ sodium citrate, $\mathrm{pH}$ 7.5. Two hundred microliters of 1-mg/ml RNase A (Qiagen, Germantown, MD) was added, and samples were incubated in a $50^{\circ} \mathrm{C}$ water bath for $1 \mathrm{~h} ; 40 \mu \mathrm{l} 20-\mathrm{mg} / \mathrm{ml}$ proteinase $\mathrm{K}$ (Roche, Basel, Switzerland) was added, and the samples were incubated an additional $1 \mathrm{~h}$ at $50^{\circ} \mathrm{C}$. After proteinase $\mathrm{K}$ treatment, 250 to $300 \mu$ l of cells was diluted to a final volume of $1 \mathrm{ml}$ in $50 \mathrm{mM}$ sodium citrate, $\mathrm{pH}$ 7.5. SYBR green I (Invitrogen, Carlsbad, CA) was present at a final dilution of $1: 500$ of the commercial stock. Samples were left at $4{ }^{\circ} \mathrm{C}$ overnight in the dark. Triton $\mathrm{X}-100$ was added to a final concentration of $0.25 \%$. The samples were sonicated using a Branson 450 Sonifier equipped with a double-step tip at an output setting of 1.5 and a duty cycle of $90 \%$ for 3 or 4 pulses. Finally, the samples were filtered, and data were acquired on a FACSCalibur (BD BioSciences, San Jose, CA).

\section{Chromosome Motion Assay}

Cell cultures were grown, induced, mounted, and then imaged on the OMX microscope as described in Pollard and Fung (2017). Imaging of live cells was performed between 14 and 24 hours after induction, generally using a 4 to $5 \mu \mathrm{m} z$-stack with sections at $0.2 \mu \mathrm{m}$ intervals, exposures of $5 \mathrm{~ms}$, and time points every $400 \mathrm{~ms}$. Bleaching of the GFP fluorophore under these conditions usually limited time course length to a maximum of 10 minutes. Image data was visualized and processed by deconvolution and denoising with algorithms embedded in the Priism imaging analysis platform (http://msg.ucsf.edu/IVE/). Chromosome contours were semi-automatically generated using MATLAB routines and algorithms. Each contour was generated via a process in which the position of a synaptonemal complex at each time point was automatically digitized, its movement then tracked through space and time, and kinetic parameters of the motion then quantified. A combination of 20 different MATLAB programs were developed expressly for this purpose (TZEOS package). For motion metrics of SC ends, an approximate equal number of minutes were evaluated for each comparison strains using 4 cells for zip3 and 11 cells for t/c1-11 zip3. 


\section{Meiotic chromosome spreads}

For pachytene spreads, 2 ml BR1919-8B strains were grown in YPD for 20-24 hours and diluted 1:3 fold for grown for 7 hours before transferring cell pellet from $1 \mathrm{ml}$ of culture into $10 \mathrm{ml}$ of $1 \%$ potassium acetate to induce sporulation at $30^{\circ} \mathrm{C}$ at $250 \mathrm{rpm}$. Cultures were spun down after 17, 18.5 and 20 hours of sporulation and resuspended in $2 \%$ potassium acetate $1 \mathrm{M}$ sorbitol with $10 \mathrm{ul} 1 \mathrm{M}$ DTT and $10 \mathrm{mM}$ zymolyase $100 \mathrm{~T}$ and incubated at $30^{\circ} \mathrm{C}$ for 26 minutes. The spheroplasted cells were gently pelleted and resuspended in $200 \mathrm{mM}$ MES + 1M sorbitol pH 6.4 and repelleted. After supernatant removal, the pellet was resuspended with 200 ul 200 mM MES followed immediately with $400-500$ ul of $37 \%$ formaldehyde. The cell suspension was divided between 3 slides. A 22x50 coverslip was placed over each slide after 10 minutes. After 30-45 minutes, the slides were washed with $4 \%$ Photoflow (Kodak, Rochester, NY) and dried. Slides were stained with primary antibodies guinea pig anti-Rap1 (1:100) and anti-rabbit Zip1 (1:50) diluted in 20\% fetal bovine serum (FBS) in 1X PBS for two hours. After two 10-minute PBS washes, 200 ul of a dilution of 1:200 of anti-guinea pig AlexaFluor 546 and anti-rabbit AlexaFluor 488 secondary antibodies (Invitrogen) in 20\% FBS in PBS were added to the slides and incubated for two hours. After two PBS washes, slides were dried briefly and mounted in $1.5 \mu \mathrm{g} / \mathrm{ml} \mathrm{DAPI}, 1 \mathrm{mg} / \mathrm{ml}$ p-phenylenediamine in PBSbuffered glycerol. Images were collected on a DeltaVision microscope (GE Healthcare, Marlborough, MA).

\section{Pairing Assay}

The one spot two spot assay was used to measure pairing in SK1 strains with Chromosome V (CEN) or Chromosome IV (TEL) marked with 256 repeats of the LacO binding site. Lacl-GFP was expressed from the CYC1 promoter from the URA3 locus, ura3-1::pCYC1-GFPlacl::URA3. To induce synchronous meiosis, diploid strains were pre-inoculated at OD600 $=0.3$ in BYTA medium..Samples were collected hourly for 8 hours. At each time point, cells were harvested, washed, pelleted and resuspended in $100 \mathrm{ml}$ of $4 \%$ paraformaldehyde, $3.4 \%$ sucrose, and held at room temperature for 10 min. Cells were washed once in $1 \mathrm{ml}$ of $0.1 \mathrm{M}$ potassium phosphate, $1.2 \mathrm{M}$ sorbitol buffer and resuspended in the same buffer. Cells were sonicated before microscopy, and spun onto concanavaline-A coated slides before being mounted with Vectashield plus DAPI. Images were collected on a Deltavision using a 60x objective and images 
were analysed using Fiji and CellProfiler. The number of GFP foci/nucleus was averaged from $~ 1000$ nuclei/timepoint)

\section{Synapsis Progression}

Cell cultures were grown, induced, mounted, and then imaged on the OMX microscope as described in Pollard and Fung (2017). Imaging of live cells was performed beginning after 5.5 hours post induction, in $10 \mu \mathrm{m}$ z-stacks with sections at 0.2 $\mu \mathrm{m}$ intervals with exposures of $5 \mathrm{~ms}$, and time interval every 30 minutes. Time courses were routinely terminated after 150 hours of imaging (time points were generally acquired with stepped-down frequency at later times: 1, 2 or 4 hour intervals). Image data was visualize, denoised and enhanced using Priism software (http://msg.ucsf.edu/IVE). Individual cells were both manually and algorithmically tracked and scored for progression past: 1) the start of Zip1 expression, 2) the onset of synapsis, 3) the termination of synapsis, and 4) the formation of spores and/or the appearance of auto-fluorescence in spores. Scripts written using MATLAB software (MathWorks Inc., Natick, MA) were utilized to analyze population progression data statistically.

\section{Whole-Genome Microarray and Next Generation Sequencing Recombination \\ Mapping}

DNA was prepared for Illumina sequencing using a NextFlex kit (BIOO Scientific, Austin TX) with Illumina-compatible indices or as described (Anderson et al., 2011) with 4-base or 8-base inline barcodes. Read alignment, genotyping and recombination mapping were performed using the ReCombine package (Anderson et al., 2011). While running CrossOver.py, the input values for 'closeCOs', 'closeNcoSame' and 'closeNCODiff' were all set to 0 . Insertions and deletions were removed from the set of genotyped markers. Recombination events within $5 \mathrm{~kb}$ of each other were then merged into single events and categorized into seven types as described (Oke et al., 2014). Gamma distributions and CoC were calculated as described (Anderson et al., 2015). Standard error for $\mathrm{CoC}$ was calculated from the average of the $25 \mathrm{~kb}$ bins used to calculate interference. S96 microarrays (Affymetrix, Santa Clara, CA) were prepared and analyzed with Allelescan software.

\section{Microarray Detection of ssDNA}

A total of $1.5 \mathrm{mg}$ each of $0 \mathrm{hr}$ and 3 or $5 \mathrm{hr}$ ssDNA samples were labeled with Cy3dUTP or Cy5-dUTP (GE Healthcare) by random priming without denaturation with $4 \mathrm{mg}$ 
random nonamer oligo (Integrated DNA Technologies, Skoie IL) and 10 units of Klenow (New England Biolabs, Ipswich, MA). Unincorporated dye was removed with Microcon columns (30 kDa MW cutoff; Millipore, Burlington, MA), and samples were cohybridized to custom Agilent arrays in accordance with a standard protocol. For each set of experiments, a dye swap was performed.

\section{Southern Blotting Analysis for DSB Measurement}

To induce synchronous meiosis, diploid strains were pre-inoculated at OD600 $=0.3$ in BYTA medium (50 mM potassium phthalate, 1\% yeast extract, $2 \%$ bactotryptone, $1 \%$ potassium acetate), grown for $16 \mathrm{hr}$ at $30 \mathrm{C}$, washed twice, and resuspended at OD600 $=1.9$ in SPO medium ( $0.3 \%$ potassium acetate). Southern analysis was performed by separating DNA fragments in 1\% agarose/1X TBE gels using pulse-field electrophoresis with a 5-45 s ramp and blotted onto Hybond-XL membranes (GE Healthcare) with alkaline transfer.

\section{Quantification and Statistical Analysis}

All statistical analysis was performed using R, Prism (GraphPad) or Microsoft Excel.

\section{Data and Software Availability}

Raw sequence data from the genome-wide analysis of recombination have been deposited in the NIH Sequence Read Archive under accession number SUB4518296. Custom MATLAB and Allelescan software is available freely upon request. Recombine software is available at https://sourceforge.net/projects/recombine/files/ 


\section{References}

Anderson C.M., Chen S.Y., Dimon M.T., Oke A., DeRisi J.L., Fung, J.C. (2011)

ReCombine: a suite of programs for detection and analysis of meiotic recombination in whole-genome datasets. PLoS One 6, e25509.

Anderson, C.M., Oke, A., Yam, P., Zhuge ,T., Fung, J.C. (2015) Reduced Crossover Interference and Increased ZMM-Independent Recombination in the Absence of Tel1/ATM. PLoS Genet. 11, e1005478.

Chacón, M.R., Delivani, P., and Tolić, I.M. (2016). Meiotic Nuclear Oscillations Are Necessary to Avoid Excessive Chromosome Associations. Cell Rep. 17, 1632-1645.

Christophorou, N., Rubin, T., Bonnet, I., Piolot, T., Arnaud, M., and Huynh, J.R. (2015). Microtubule-driven nuclear rotations promote meiotic chromosome dynamics. Nat. Cell Biol. 17, 1388-1400.

Church, K. and Moens, P. (1976). Centromere behavior during interphase and meiotic prophase in Allium fistulosum from 3-D, E.M. reconstruction. 56, 249-263.

Conrad, M.N., Dominguez, A.M., and Dresser, M.E. (1997). Ndj1p, a meiotic telomere protein required for normal chromosome synapsis and segregation in yeast. Science $276,1252-1255$.

Conrad, M.N., Lee, C.-Y., Chao, G., Shinohara, M., Kosaka, H., Shinohara, a, Conchello, J., and Dresser, M.E. (2008). Rapid telomere movement in meiotic prophase is promoted by NDJ1, MPS3, and CSM4 and is modulated by recombination. Cell 133, $1175-1187$.

Ding, D.Q., Chikashige, Y., Haraguchi, T., and Hiraoka, Y. (1998). Oscillatory nuclear movement in fission yeast meiotic prophase is driven by astral microtubules, as revealed by continuous observation of chromosomes and microtubules in living cells. J. Cell Sci.

111 Pt 6, 701-712. 
Evans, S.K., and Lundblad, V. (1999). Est1 and Cdc13 as comediators of telomerase access. Science 286, 117-120.

Hass, E.P., and Zappulla, D.C. (2015). The Ku subunit of telomerase binds Sir4 to recruit telomerase to lengthen telomeres in S. cerevisiae. Elife 4, 1-19.

Hassold T. and Hunt P. (2001) To err (meiotically) is human: the genesis of human aneuploidy. Nat. Rev. Genet. 2, 280-291.

Hiraoka, Y., and Dernburg, A.F. (2009). The SUN Rises on Meiotic Chromosome Dynamics. Dev. Cell 17, 598-605.

Huet, J., Cottrelle, P., Cool, M., Vignais, M.L., Thiele, D., Marck, C., Buhler, J.M., Sentenac, A., and Fromageot, P. (1985). A general upstream binding factor for genes of the yeast translational apparatus. EMBO J. 4, 3539-3547.

Jay, K.A., Smith, D.L., and Blackburn, E. (2016). Early Loss of Telomerase Action in Yeast Creates a Dependence on the DNA Damage Response Adaptor Proteins. Mol. Cell. Biol. 36, MCB 1908-19.

Joseph, I., Dingwu, J., and Lustig, A.J. (2005). Ndj1p-Dependent Epigenetic Resetting of Telomere Size in Yeast Meiosis. Curr. Biol. 15, 231-237.

Joyce, E.F., Apostolopoulos, N., Beliveau, B.J., and Wu, C.T. (2013). Germline Progenitors Escape the Widespread Phenomenon of Homolog Pairing during Drosophila Development. PLoS Genet. 9. e1004013.

Keeney, S., Giroux, C.N., and Kleckner, N. (1997). Meiosis-specific DNA double-strand breaks are catalyzed by Spo11, a member of a widely conserved protein family. Cell 88 , 375-384. 
Kosaka, H., Shinohara, M., and Shinohara, A. (2008). Csm4-dependent telomere movement on nuclear envelope promotes meiotic recombination. PLoS Genet. 4. e1000196.

Lee CY, Horn HF, Stewart CL, Burke B, Bolcun-Filas E, Schimenti JC, Dresser ME, Pezza RJ. (2015). Mechanism and regulation of rapid telomere prophase movements in mouse meiotic chromosomes. Cell Rep. 11, 551-563.

Lin, J., Ly, H., Hussain, A., Abraham, M., Pearl, S., Tzfati, Y., Parslow, G., and Blackburn, E.H. (2004). A universal telomerase RNA core structure includes structured motifs required for binding the telomerase reverse transcriptase. $101,14713-14718$.

Lui, D.Y., Cahoon, C.K. and Burgess, S.M. (2013) Multiple opposing constraints govern chromosome interactions during meiosis. PLoS Genet. 9. e1003197.

MacQueen, A.J., and Hochwagen, A. (2011). Checkpoint mechanisms: The puppet masters of meiotic prophase. Trends Cell Biol. 21, 393-400.

MacQueen, A.J., Phillips, C.M., Bhalla, N., Weiser, P., Villeneuve, A.M., and Dernburg, A.F. (2005). Chromosome sites play dual roles to establish homologous synapsis during meiosis in C. elegans. Cell 123, 1037-1050.

Mancera, E., Bourgon, R., Huber, W., and Steinmetz, L.M. (2011). Genome-wide survey of post-meiotic segregation during yeast recombination. Genome Biol. 12. R36.

Marshall, W.F., and Fung, J.C. (2016). Modeling meiotic chromosome pairing: nuclear envelope attachment, telomere-led active random motion, and anomalous diffusion. Phys. Biol. 13, 26003.

Oke, A., Anderson, C.M., Yam, P., and Fung, J.C. (2014). Controlling meiotic recombinational repair - specifying the roles of ZMMs, Sgs1 and Mus81/Mms4 in crossover formation. PLoS Genet 10, e1004690.

Pollard M.G., and Fung J.C. (2017) In Vivo Imaging of Budding Yeast Meiosis. Methods Mol Biol. 1471, 175-186. 
Reichard P. (1988). Interactions between deoxyribonucleotide and DNA synthesis.

Annu. Rev. Biochem. 57, 349-374.

Revenkova, E., Eijpe, M., Heyting, C., Hodges, C.A., Hunt, P.A., Liebe, B., Scherthan, $H$., and Jessberger, R. (2004). Cohesin SMC1 $\beta$ is required for meiotic chromosome dynamics, sister chromatid cohesion and DNA recombination. Nat. Cell Biol. 6, 555-562.

Sato, A., Isaac, B., Phillips, C.M., Rillo, R., Carlton, P.M., Wynne, D.J., Kasad, R.A., and Dernburg, A.F. (2009). Cytoskeletal Forces Span the Nuclear Envelope to Coordinate Meiotic Chromosome Pairing and Synapsis. Cell 139, 907-919.

Scherthan Weich, S., Schwegler, H., Heyting,C., Harle, M., H., and Cremer, T. (1996). Centromere and telomeremovements during early meiotic prophase of mouse and man areassociated with the onset of pairing. J.Cell.Biol 134, 1109-1125.

Scherthan, H. (2007). Telomere attachment and clustering during meiosis. Cell. Mol. Life Sci. $64,117-124$.

Scherthan, H., Wang, H., Adelfalk, C., White, E.J., Cowan, C., Cande, W.Z., and Kaback, D.B. (2007). Chromosome mobility during meiotic prophase in Saccharomyces cerevisiae. Proc. Natl. Acad. Sci. U. S. A. 104, 16934-16939.

Schober, H., Ferreira, H., Kalck, V., Gehlen, L.R., and Gasser, S.M. (2009). Yeast telomerase and the SUN domain protein Mps3 anchor telomeres and repress subtelomeric recombination. Genes Dev. 23, 928-938.

Sheehan, M.J., and Pawlowski, W.P. (2009). Live imaging of rapid chromosome movements in meiotic prophase I in maize. Proc. Natl. Acad. Sci. U. S. A. 106, 2098920994.

Sun H., Treco, D., and Szostak, J.W. (1991) Extensive 3'-overhanging, single-stranded DNA associated with the meiosis-specific double-strand breaks at the ARG4 recombination initiation site. Cell 11, 6328-6336. 
Sym M., Englebrecht, J.A. and Roeder, G.S. (1993) ZIP1 is a synaptonemal complex protein required for meiotic chromosome synapsis. $72,365-378$.

Taddei, A., Hediger, F., Neumann, F.R., Bauer, C., and Gasser, S.M. (2004). Separation of silencing from perinuclear anchoring functions in yeast Ku80, Sir4 and Esc1 proteins. EMBO J. 23, 1301-1312.

Thacker, D., Mohibullah, N., Zhu, X., and Keeney, S. (2014). Homologue engagement controls meiotic DNA break number and distribution. Nature 510, 241-246.

Trelles-Sticken, E., Adelfalk, C., Loidl, J., and Scherthan, H. (2005). Meiotic telomere clustering requires actin for its formation and cohesin for its resolution. J. Cell Biol. 170, 213-223.

Trelles-Sticken, E., Dresser, M.E., and Scherthan, H. (2000). Meiotic telomere protein $\mathrm{Ndj} 1 \mathrm{p}$ is required for meiosis-specific telomere distribution, bouquet formation and efficient homologue pairing. J. Cell Biol. 151, 95-106.

Tsubouchi T. and Roeder, G.S. (2005). A synaptonemal complex protein promotes homology-independent centromere coupling. Science 308, 870-873.

Uhlmann, F., and Nasmyth, K. (1998). Cohesion between sister chromatids must be established during DNA replication. Curr. Biol. 8, 1095-1102.

Wanat, J.J., Kim, K.P., Koszul, R., Zanders, S., Weiner, B., Kleckner, N., and Alani, E. (2008). Csm4, in collaboration with Ndj1, mediates telomere-led chromosome dynamics and recombination during yeast meiosis. PLoS Genet. 4, e1000188.

Wu, H., and Burgess, S.M. (2006). Promotes meiotic recombination in budding yeast Ndj1, a telomere-associated protein, Promotes Meiotic Recombination in Budding Yeast. 26, 3683-3694. 
Wynne, D.J., Rog, O., Carlton, P.M., and Dernburg, A.F. (2012). Dynein-dependent processive chromosome motions promote homologous pairing in C. elegans meiosis. J. Cell Biol. 196, 47-64.

Xie, Z., Jay, K.A., Smith, D.L., Zhang, Y., Liu, Z., Zheng, J., Tian, R., Li, H., and Blackburn, E.H. (2015). Early telomerase inactivation accelerates aging independently of telomere length. Cell 160, 928-939.

Zickler, D. (2006). From early homologue recognition to synaptonemal complex formation. Chromosoma 115, 158-174.

Zickler, D., and Kleckner, N. (2015). Recombination, Pairing and Synapsis of Homologs during Meiosis. Cold Spring Harb. Perspect Biol 7, a016626.

\section{ACKNOWLEDGEMENTS}

Support is provided by R01 GM116895 to AO, TG, WM \& JCF; RO1 GM026259 to DLS

\& EHB R01 GM111715 to AH \& NJP and R01 GM075119 to SB \& DC. 


\section{Supplemental Tables and Figures}

Supplemental Table S1. Yeast strains

\begin{tabular}{|c|c|c|c|}
\hline Strain names & $\begin{array}{l}\text { Strain } \\
\text { number(s) }\end{array}$ & $\begin{array}{l}\text { Strain } \\
\text { background }\end{array}$ & Relevant genotype \\
\hline wt BR1919-8B & $\begin{array}{l}\text { yEHB5- } \\
1055 / 5-1056\end{array}$ & BR1919 & $M A T a / \alpha$ \\
\hline $\operatorname{sir} 4 \Delta$ & $\begin{array}{l}\text { yEHB5-1411/ } \\
1412\end{array}$ & BR1919 & $M A T a / \alpha \operatorname{sir} 4 \Delta:: N A T / \operatorname{sir} 4 \Delta:: N A T$ \\
\hline esc1s & $\begin{array}{l}\text { yEHB5-1564/ } \\
1565\end{array}$ & BR1919 & MATa/ $\alpha$ esc1 $1 \triangle:: N A T / e s c 1 \Delta:: N A T$ \\
\hline ku80s & JS472/JS474 & BR1919 & 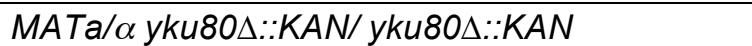 \\
\hline t/c1s & yEHB1604 & BR1919 & $M A T a / \alpha$ t/c1 $1 \Delta: N A T / t / c 1 \Delta \because: N A T$ \\
\hline t/c1 $1 \Delta p T L C 1$ & $\begin{array}{l}\text { yEHB5800/ } \\
5794\end{array}$ & BR1919 & 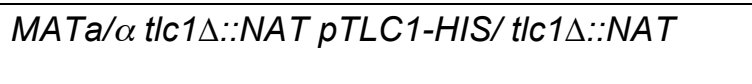 \\
\hline t/c1-11 & $\begin{array}{l}\text { yEHB5-1338/ } \\
1339\end{array}$ & BR1919 & MATa/ $\alpha$ TLC1::t/c1-11/TLC1::t/c1-11 \\
\hline t/c1s pt/c1-11 & $\begin{array}{l}\text { yEHB5796/ } \\
5798\end{array}$ & BR1919 & 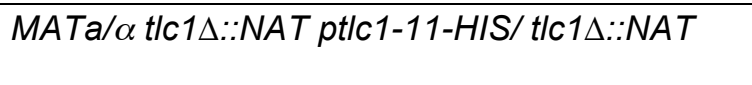 \\
\hline t/c1-101 & $\begin{array}{l}\text { yEHB5-1340/ } \\
1341\end{array}$ & BR1919 & MATa/ $\alpha$ TLC1::t/c1-101/ TLC1::t/c1-101 \\
\hline$t / c 1 \Delta p t / c 1-101$ & yEHB5895 & BR1919 & 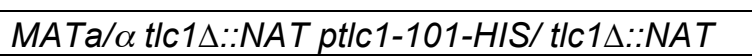 \\
\hline t/c1s long & yEHB1607 & BR1919 & $\begin{array}{l}\text { MATa/ } \alpha \text { tlc1 } 1:: N A T / \text { tlc1 } 1:: N A T \text { pTLC1-HIS } \\
\text { pCdc13-Est2-LEU for lengthening only }\end{array}$ \\
\hline t/c1-11 long & yEHB1612 & BR1919 & $\begin{array}{l}\text { MATa/ } \alpha \text { t/c1-11 / t/c1-11 pTLC1-HIS } \\
\text { pCdc13-Est2-LEU for lengthening only }\end{array}$ \\
\hline$s m / 1 \Delta$ & $\begin{array}{l}\text { yEHB5-1504/ } \\
1505\end{array}$ & BR1919 & $M A T a / \alpha$ sml1 $\because: N A T / s m / 1 \Delta:: N A T$ \\
\hline$s m / 1 \Delta t / c 1-11$ & $\begin{array}{l}\text { yEHB5-1508/ } \\
5109\end{array}$ & BR1919 & 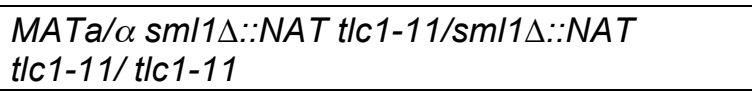 \\
\hline$s m / 1 \Delta t / c 1-101$ & $\begin{array}{l}\text { yEHB5-1512/ } \\
1513\end{array}$ & BR1919 & 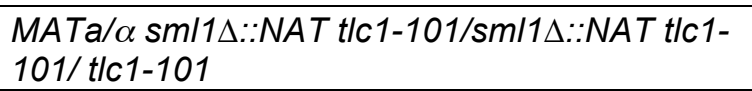 \\
\hline rif2 & $\begin{array}{l}\text { yEHB6015/ } \\
6016\end{array}$ & BR1919 & MATa/ $\alpha$ rif2 $\triangle:: T R P /$ rif2 $\triangle:: T R P$ \\
\hline $\operatorname{csm} 4$ & $\begin{array}{l}\text { yEHB5-1567/ } \\
5-1568\end{array}$ & BR1919 & $M A T a / \alpha \operatorname{csm} 4 \Delta:: K A N / \operatorname{csm} 4 \Delta:: K A N$ \\
\hline$n d j 1$ & $\begin{array}{l}\text { yEHB6015/ } \\
6016\end{array}$ & BR1919 & MATa/ $\alpha$ ndj1 $1:: T R P / n d j 1 \Delta:: T R P$ \\
\hline $\operatorname{sir} 2 \Delta$ & yEHB5-1370 & BR1919 & $M A T a / \alpha$ sir2 $2:: N A T / \operatorname{sir} 2 \Delta \because: N A T$ \\
\hline dot1s & yEHB5-1378 & BR1919 & $M A T a / \alpha$ dot1 $\because: N A T / d o t 1 \Delta \because: N A T$ \\
\hline $\begin{array}{l}\text { zip3 } 2 \text { ZIP1- } \\
\text { GFP }\end{array}$ & $\begin{array}{l}\text { yEHB5-1204/ } \\
1205\end{array}$ & BR1919 & 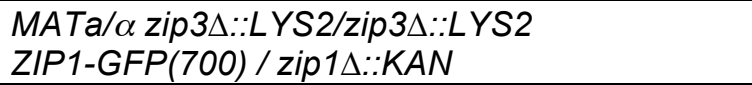 \\
\hline $\begin{array}{l}\text { tlc1-11 zip3s } \\
\text { ZIP1-GFP }\end{array}$ & $\begin{array}{l}\text { yEHB5-1206/ } \\
1207\end{array}$ & BR1919 & $\begin{array}{l}\text { MATa/ } \alpha \text { tlc1-11/tlc1-11, zip3::LYS2 /zip3::LYS2, } \\
\text { ZIP1-GFP(700)/zip1A::KAN }\end{array}$ \\
\hline $\begin{array}{l}\text { t/c1-101 zip3s } \\
\text { ZIP1-GFP }\end{array}$ & $\begin{array}{l}\text { yEHB5-1208/ } \\
1209\end{array}$ & BR1919 & $\begin{array}{l}\text { MATa/ } \alpha, \text { tlc1-101/tlc1-101, zip3::LYS2 } \\
\text { /zip3::LYS2, ZIP1-GFP(700)/zip1A::KAN }\end{array}$ \\
\hline wt S96 & JCF1100 & S96a & \\
\hline wt YJM789 & JCF4411 & YJM789 $\alpha$ & \\
\hline t/c1-11 S96 & yEHB5680 & S96a & tlc1s pt/c1-11 \\
\hline $\begin{array}{l}\text { tlc1-11 } \\
\text { YJM789 }\end{array}$ & yEHB5681 & YJM789 $\alpha$ & t/c1s pt/c1-11 \\
\hline tlc1-11 S96 & yEHB5680 & S96a & TLC1::t/c1-11 \\
\hline $\begin{array}{l}\text { tlc1-11 } \\
\text { YJM789 }\end{array}$ & yEHB5681 & YJM789 $\alpha$ & TLC1::t/c1-11 \\
\hline
\end{tabular}




\begin{tabular}{|c|c|c|c|}
\hline t/c1-101 S96 & yEHB5680 & S96a & t/c1s pt/c1-101 \\
\hline $\begin{array}{l}\text { tlc1-101 } \\
\text { YJM789 }\end{array}$ & yEHB5681 & YJM789 $\alpha$ & t/c1s pt/c1-101 \\
\hline t/c1-101 S96 & yEHB5682 & S96a & TLC1::t/c1-101 \\
\hline $\begin{array}{l}\text { t/c1-101 } \\
\text { YJM789 }\end{array}$ & yEHB5683 & YJM789 $\alpha$ & TLC1::t/c1-101 \\
\hline ku80 $\mathrm{S} 96$ & yEHB5740 & S96a & 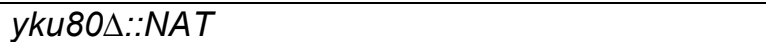 \\
\hline $\begin{array}{l}\text { ku80D } \\
\text { YJM789 }\end{array}$ & yEHB5741 & YJM789 $\alpha$ & 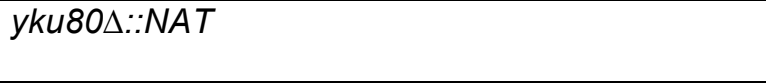 \\
\hline long telo S96 & yEHB23001 & S96a & cdc13::Cdc13-Est2-URA3->loop-out \\
\hline $\begin{array}{l}\text { long telo } \\
\text { YJM789 }\end{array}$ & yEHB23002 & YJM789 $\alpha$ & cdc13::Cdc13-Est2-URA3->loop-out \\
\hline $\begin{array}{l}\text { long telo est2 } \\
\text { S96 }\end{array}$ & yEHB5816 & S96a & cdc13::Cdc13-Est2-URA3->loop-out est2A::NAT \\
\hline $\begin{array}{l}\text { long telo est2 } \\
\text { YJM789 }\end{array}$ & yEHB5831 & YJM789 $\alpha$ & cdc13::Cdc13-Est2-URA->loop-out 3 est2 $\triangle:: N A T$ \\
\hline $\begin{array}{l}\text { long telo t/c1- } \\
11 \text { S96 }\end{array}$ & yEHB5-1220 & S96a & cdc13::Cdc13-Est2-URA3->loop-out tlc1-11 \\
\hline $\begin{array}{l}\text { long telo t/c1- } \\
11 \text { YJM789 }\end{array}$ & yEHB-1224 & YJM789 $\alpha$ & cdc13::Cdc13-Est2-URA3->loop-out tlc1-11 \\
\hline$s m / 1 \Delta$ S96 & yEHB5-1501 & S96a & $s m / 1 \Delta:: N A T$ \\
\hline $\begin{array}{l}s m / 1 \Delta \\
\text { YJM789 }\end{array}$ & yEHB5-1502 & YJM789 $\alpha$ & $s m / 1 \Delta:: N A T$ \\
\hline $\begin{array}{l}\text { t/c1-11 sml1s } \\
\text { s96 }\end{array}$ & yEHB5-1555 & S96a & TLC1::tlc1-11 sml1A::NAT \\
\hline $\begin{array}{l}\text { t/c1-11 sml1s } \\
\text { YJM789 }\end{array}$ & yEHB5-1560 & YJM789 $\alpha$ & TLC1::tlc1-11 sml1A::NAT \\
\hline esc1 $1 \Delta$ S96 & $\mathrm{H} 6590$ & S96a & esc1s::hphMX4 \\
\hline $\begin{array}{l}\text { esc1s } \\
\text { YJM789 }\end{array}$ & H6591 & YJM789 $\alpha$ & esc1 $1::$ hphMX4 \\
\hline sir4A S96 & yEHB5881 & S96a & $\operatorname{sir} 4 \Delta:: N A T$ \\
\hline sir4A YJM789 & yEHB5883 & YJM789 $\alpha$ & $\operatorname{sir} 4 \triangle \because: N A T$ \\
\hline$n d j 1 \Delta \mathrm{S} 96$ & SYC1112 & S96a & $n d j 1 \triangle:: K A N$ \\
\hline ndj1 $1 \Delta$ YJM789 & SYC1113 & YJM789 $\alpha$ & $n d j 1 \Delta:: K A N$ \\
\hline $\operatorname{csm} 4 \Delta \mathrm{S} 96$ & PY379 & S96a & $\operatorname{csm} 4 \Delta:: K A N$ \\
\hline $\begin{array}{l}\operatorname{csm} 4 \Delta \\
\text { YJM789 }\end{array}$ & PY730 & YJM789 $\alpha$ & $\operatorname{csm} 4 \Delta:: K A N$ \\
\hline tel1 $\mathrm{S}$ S96 & yCA703 & S96a & tel1 $1:: K A N$ \\
\hline 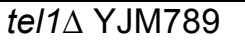 & yCA704 & YJM789 $\alpha$ & tel1 $\triangle:: K A N$ \\
\hline sgs1 $1 \Delta 96$ & SYC1120 & S96a & $\operatorname{sgs} 1 \Delta:: K A N$ \\
\hline $\begin{array}{l}\text { sgs14 } \\
\text { YJM789 }\end{array}$ & SYC1121 & YJM789 $\alpha$ & $\operatorname{sgs} 1 \Delta:: K A N$ \\
\hline$m s h 4 \Delta$ S96 & SYC1110 & S96a & $m s h 4 \Delta:: K A N$ \\
\hline $\begin{array}{l}m s h 4 \Delta \\
\text { YJM789 }\end{array}$ & SYC1112 & YJM789 $\alpha$ & $m s h 4 \Delta:: K A N$ \\
\hline wt & $\begin{array}{l}\text { yEHB5-1074/ } \\
1075\end{array}$ & SK1 & $M A T a / \alpha$ \\
\hline t/c1-11 & $\begin{array}{l}\text { yEHB5-1192/ } \\
1193\end{array}$ & SK1 & MATa/ $\alpha$ TLC1::t/c1-11/TLC1::t/c1-11 \\
\hline t/c1-101 & $\begin{array}{l}\text { yEHB5-1165/ } \\
1181\end{array}$ & SK1 & MATa/ $\alpha$ TLC1::t/c1-101/TLC1::t/c1-101 \\
\hline kus & $\begin{array}{l}\text { yEHB5-1252/ } \\
1255\end{array}$ & SK1 & 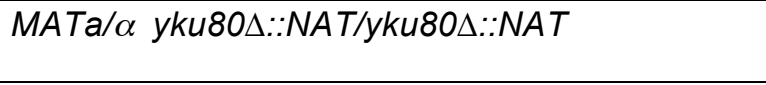 \\
\hline$n d j 1 \Delta$ & yEHB5-1332/ & SK1 & MATa/a $\Delta n d j 1:: H p h B / \Delta n d j 1:: H p h B$ \\
\hline
\end{tabular}




\begin{tabular}{|c|c|c|c|}
\hline & 1333 & & \\
\hline $\begin{array}{l}\text { wt (DSB, } \\
d m c 1 \Delta)\end{array}$ & $\begin{array}{l}\text { yEHB5894/ } \\
5893\end{array}$ & SK1 & $\begin{array}{l}\text { MAT a/ } \alpha \text { arg } 4-N S P \text { dmc1 } 1:: A R G 4 / a r g 4-N S P \\
d m c 1 \Delta:: A R G 4\end{array}$ \\
\hline $\begin{array}{l}\text { t/c1-11 } \\
(\mathrm{DSB}, d m c 1 \Delta)\end{array}$ & $\begin{array}{l}\text { yEHB5-1154/ } \\
1151\end{array}$ & SK1 & $\begin{array}{l}\text { MAT a/ } \alpha \text { tlc1-11/tlc1-11 dmc1 } \because:: A R G 4 / \\
d m c 1 \Delta:: A R G 4\end{array}$ \\
\hline $\begin{array}{l}\text { t/c1-101 } \\
(\mathrm{DSB}, d m c 1 \Delta)\end{array}$ & $\begin{array}{l}\text { yEHB5-1066/ } \\
1062\end{array}$ & SK1 & $\begin{array}{l}\text { MAT a/ } \alpha \text { t/c1-101/t/c1-101dmc1A::ARG4/ } \\
d m c 1 \Delta:: A R G 4\end{array}$ \\
\hline $\begin{array}{l}\text { wt } \\
\text { (DSB, DMC1) }\end{array}$ & $\begin{array}{l}\text { yEHB5-1216 } \\
1219\end{array}$ & SK1 & 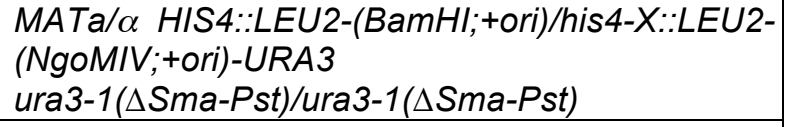 \\
\hline $\begin{array}{l}\text { t/c1-11 } \\
(\mathrm{DSB}, \mathrm{DMC} 1)\end{array}$ & $\begin{array}{l}\text { yEHB5-1237/ } \\
1246\end{array}$ & SK1 & 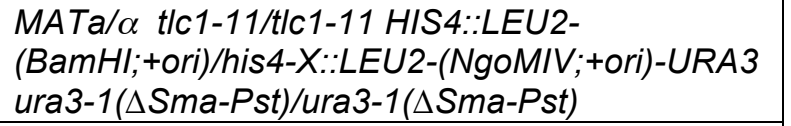 \\
\hline $\begin{array}{l}\text { t/c1-101 } \\
\text { (DSB, DMC1) }\end{array}$ & $\begin{array}{l}\text { yEHB5-1238/ } \\
1245\end{array}$ & SK1 & 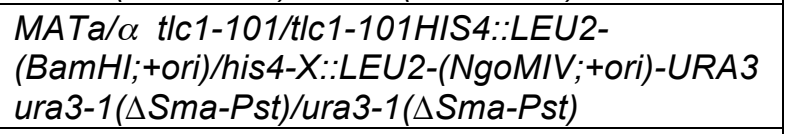 \\
\hline $\begin{array}{l}\text { wt } \\
\text { (pairing CEN) }\end{array}$ & $\begin{array}{l}\text { yEHB5-1539/ } \\
1533\end{array}$ & SK1 & $\begin{array}{l}\text { MATa/ } \alpha \text { ura3-1::pCYC1-GFPlacl::URA3/ } \\
\text { ura3-1::pCYC1-GFPlacl::URA3 } \\
\text { cenV::IacO::NAT/cenV::IacO::NAT }\end{array}$ \\
\hline $\begin{array}{l}\text { t/c1-11 } \\
\text { (pairing CEN) }\end{array}$ & $\begin{array}{l}\text { yEHB5-1542/ } \\
1537\end{array}$ & SK1 & $\begin{array}{l}\text { MATa/ } \alpha \text { t/c1-11/tlc1-11 } \\
\text { GFPlacl::URA3/GFPlacl::URA3 } \\
\text { cenV::IacO::NAT/cenV::IacO::NAT }\end{array}$ \\
\hline $\begin{array}{l}\text { t/c1-101 } \\
\text { (pairing CEN) }\end{array}$ & $\begin{array}{l}\text { yEHB5-1551/ } \\
1553\end{array}$ & SK1 & $\begin{array}{l}\text { MATa/ } \alpha \text { t/c1-101/tlc1-101 } \\
\text { GFPlacl::URA3/GFPlacl::URA3 } \\
\text { cenV::IacO::NAT/cenV::IacO::NAT }\end{array}$ \\
\hline $\begin{array}{l}\text { wt } \\
\text { (pairing TEL) }\end{array}$ & $\begin{array}{l}\text { yEHB5-1384/ } \\
1381\end{array}$ & SK1 & 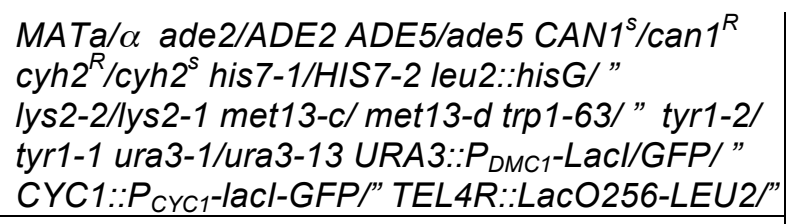 \\
\hline $\begin{array}{l}\text { t/c1-11 } \\
\text { (pairing TEL) }\end{array}$ & $\begin{array}{l}\text { yEHB5-1389/ } \\
1391\end{array}$ & SK1 & $\begin{array}{l}\text { MATa/ } \alpha \text { yEHB5-1384 /yEHB5-1381 } \\
\text { t/c1-11/tIc1-11 }\end{array}$ \\
\hline $\begin{array}{l}\text { t/c1-101 } \\
\text { (pairing TEL) }\end{array}$ & $\begin{array}{l}\text { yEHB5-1393/ } \\
1395\end{array}$ & SK1 & $\begin{array}{l}\text { MATa/ } \alpha \text { yEHB5-1384 /yEHB5-1381 } \\
\text { t/c1-101/t/c1-101 }\end{array}$ \\
\hline
\end{tabular}

The genotype of all BR1919-8B strains is leu2-3,122 his4-260 usa3-1 ade2-1 thr1-4lys2 trp1-289, plus additional features listed below. BR1919 diploids are fully homozygous at all loci except MAT and were used for imaging experiments and for measuring spore viability.

S96 and YJM789 haploid parents were used to make hybrid diploids for recombination mapping. The genotypes for S96 strains is MATa ho lys5 and for YJM789 strains is MAT $\alpha$ ho::hisG lys2 cyh, plus the additional features listed below.

The SK1 strain background was used for time course experiments requiring meiotic synchrony. The SK1 genotype for chromosomal and whole genome DSB measurement is lys2 ho::LYS2 leu2::hisG his3::hisG trp1::hisG ura3-1 with modifications listed below.

Strain acknowledgements: Gloria Brar (wt SK1), Michael Dresser (SK1 TEL4R pairing) and Brad Stohr (early steps to creating hybrid long telomere strain). Plasmid acknowledgements: Susan Gasser, pSIR4-2 $\mu$-URA (pSG237); Adam Rudner, pRS313-SIR2-HIS (pAR455) and Kyle Jay, pRS406-tlc1-11 (pEHB30,006) and pRS406-tlc1-101 (pEHB30,007). 
Table S2. Spore viability

\begin{tabular}{|c|c|c|c|c|c|c|c|}
\hline $\begin{array}{l}\% \text { Viable } \\
\text { spores / tetrad }\end{array}$ & 0 & 1 & 2 & 3 & 4 & $\begin{array}{l}\text { Total } \\
\text { Viability }\end{array}$ & Notes \\
\hline 1919 & 1 & 0 & 2.5 & 12.5 & 84 & 94.4 & WT used for microscopy \\
\hline $\begin{array}{l}\text { S96xYJM789 } \\
(S \times Y)\end{array}$ & 1 & 0 & 10 & 15 & 74 & 90 & $\begin{array}{l}\text { WT used for sequencing and } \mathrm{CO} \\
\text { analysis }\end{array}$ \\
\hline S288C & 0.4 & 0.4 & 3.4 & 5.2 & 90.4 & 98.2 & WT standard lab strain, \\
\hline ptlc1-11 (SxY) & 13.5 & 17.5 & 29 & 33 & 7 & 49 & Plasmid-t/c1-11 covering $t / c 1 \Delta$ \\
\hline pt/c1-11 (1919) & 23.7 & 14.6 & 24.1 & 18.3 & 18.3 & 48 & Plasmid-t/c1-11 covering $t / c 1 \Delta$ \\
\hline $\begin{array}{l}\text { ptlc1- } \\
11+R a p 1(S x Y)\end{array}$ & 2.2 & 5.4 & 15.5 & 28.6 & 48 & 78.8 & 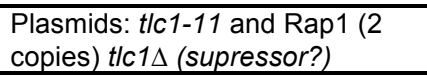 \\
\hline $\begin{array}{l}\text { ptlc1- } \\
11+\operatorname{Rap1}\left(S_{X} Y\right)\end{array}$ & 82.5 & 12.5 & 4.5 & 0 & 0 & 5.6 & $\begin{array}{l}\text { Plasmids: t/c1-11 and Rap1 (1 } \\
\text { copy) t/c1 }\end{array}$ \\
\hline tlc1-11 (1919) & 0.4 & 2 & 13.8 & 25.6 & 58.5 & 76.8 & Mutation integrated at TLC1 \\
\hline pt/c1-101 (SxY) & 11.5 & 12 & 15.5 & 20.5 & 40 & 67 & Plasmid-t/c1-101 covering $t / c 1 \Delta$ \\
\hline t/c1-101 (1919) & 0 & 0.75 & 5.6 & 23 & 70.5 & 83 & Mutation integrated at TLC1 \\
\hline yKu80s (1919) & 0 & 0 & 5.9 & 12.8 & 81.9 & 94 & Deletion, marked with KAN \\
\hline$y K u 80 \Delta(S x Y)$ & 0 & 1 & 15 & 19.5 & 64.5 & 87 & Deletion, marked with KAN \\
\hline long telo (1919) & 1 & 2 & 5 & 30 & 62 & 87.5 & pCdc13-Est2 \\
\hline long telo $(S x Y)$ & 0.5 & 3.5 & 14.5 & 24 & 58 & 84.2 & pCdc13-Est2 \\
\hline $\begin{array}{l}\text { long telo } \Delta \text { est2 } \\
(S X Y)\end{array}$ & 0.4 & 3.6 & 18.8 & 19.6 & 57.6 & 82 & pCdc13-Est2 \\
\hline $\begin{array}{l}\text { Cdc13-T308A } \\
(S x Y)\end{array}$ & 1.5 & 1.5 & 6 & 16.5 & 74.55 & 90.4 & Less telomerase recruitment \\
\hline $\operatorname{zip2}^{*}(S x Y)$ & 20.6 & 18.8 & 28.5 & 16.7 & 15.5 & 46.9 & control \\
\hline $\begin{array}{l}\text { est2A (S288C) } 50 \\
\text { gen }\end{array}$ & 23 & 26 & 27 & 17 & 7 & 40 & Without EST2 for 50 generations \\
\hline $\begin{array}{l}\text { est2-D530A } \\
\text { (S288C) } 50 \text { gen }\end{array}$ & 3 & 7.5 & 15 & 34.5 & 40 & 75.5 & $\begin{array}{l}\text { Catalytically dead EST2 for } 50 \\
\text { generations }\end{array}$ \\
\hline $\begin{array}{l}\text { est2A (S288C) } 30 \\
\text { gen }\end{array}$ & 1.3 & 0.6 & 2 & 10 & 85.3 & 95 & Without EST2 for 30 generations \\
\hline $\begin{array}{l}\text { est2-D530A } \\
\text { (S288C) } 30 \text { gen }\end{array}$ & 1 & 6 & 18 & 27 & 48 & 79 & $\begin{array}{l}\text { Catalytically dead EST2 for } 30 \\
\text { generations }\end{array}$ \\
\hline $\begin{array}{l}\text { t/c1 } 1 \Delta(S 288 C) 30 \\
\text { gen }\end{array}$ & 1.5 & 1 & 8.5 & 24.5 & 64.5 & 88 & Without TLC1 for 30 generations \\
\hline $\begin{array}{l}\text { est2-D530A } \Delta s \mathrm{ml1} \\
\text { (S288C) } 30 \mathrm{gen}\end{array}$ & 0.5 & 4 & 9 & 33 & 53.5 & 84 & $\begin{array}{l}\text { Catalytically dead EST2 for } 30 \\
\text { generations, sml1 }\end{array}$ \\
\hline $\begin{array}{l}\text { t/c1 } 1 \Delta \mathrm{sm} / 1 \Delta \\
(\mathrm{S} 288 \mathrm{C}) 30 \text { gen }\end{array}$ & 0 & 1.5 & 13.5 & 23.5 & 61.5 & 87 & $\begin{array}{l}\text { Without TLC1 for } 30 \text { generations, } \\
s m / 1 \Delta\end{array}$ \\
\hline $\operatorname{sir} 2 \Delta(1919)$ & 5 & 1.2 & 6.2 & 23.7 & 63.7 & 85 & \\
\hline $\operatorname{sir} 2 \Delta(S x Y)$ & 4.5 & 3.5 & 11 & 11.5 & 69.5 & 84.5 & \\
\hline $\operatorname{sir} 4 \Delta(1919)$ & 0 & 0 & 2 & 14 & 84 & 95 & \\
\hline $\operatorname{sir} 4 \Delta(S x Y)$ & 1 & 1 & 7 & 10.5 & 80.5 & 92 & \\
\hline $\operatorname{dot1} \Delta(1919)$ & 0.5 & 1 & 4 & 13 & 81.5 & 93.5 & \\
\hline $\operatorname{dot} 1 \Delta(S x Y)$ & 6 & 2 & 17.5 & 16.5 & 58 & 78 & Note strain background difference \\
\hline $\operatorname{esc} 1 \Delta(1919)$ & 0 & 0.4 & 4.5 & 11.3 & 83.6 & 94.5 & \\
\hline $\begin{array}{l}\text { zip3s zip1-GFP } \\
\text { (!919) }\end{array}$ & 1.6 & 5 & 19.1 & 20 & 54.1 & 80 & Motion background \\
\hline $\begin{array}{l}\text { tlc1-11 zip3s zip1- } \\
\text { GFP (1919) }\end{array}$ & 8.3 & 3.3 & 20 & 24.1 & 44.1 & 73 & $\begin{array}{l}\text { Motion background, 4-spore } \\
\text { decrease, } 0 \text {-spore increase }\end{array}$ \\
\hline $\begin{array}{l}\text { tlc1-101 zip3s } \\
\text { zip1-GFP (1919) }\end{array}$ & 9.1 & 5.8 & 19.1 & 19.1 & 46.6 & 72 & $\begin{array}{l}\text { Motion background, } 4 \text {-spore } \\
\text { decrease, } 0 \text {-spore increase }\end{array}$ \\
\hline rif2 & 1.6 & 4 & 12 & 31 & 51 & 81.5 & \\
\hline $\operatorname{csm} 4 \Delta(S x Y)$ & 56 & 18.5 & 10.5 & 6.5 & 8 & 24.35 & \\
\hline csm4 4 (1919) & 26 & 5 & 20 & 9.5 & 39.5 & 45.9 & \\
\hline
\end{tabular}




\section{A}
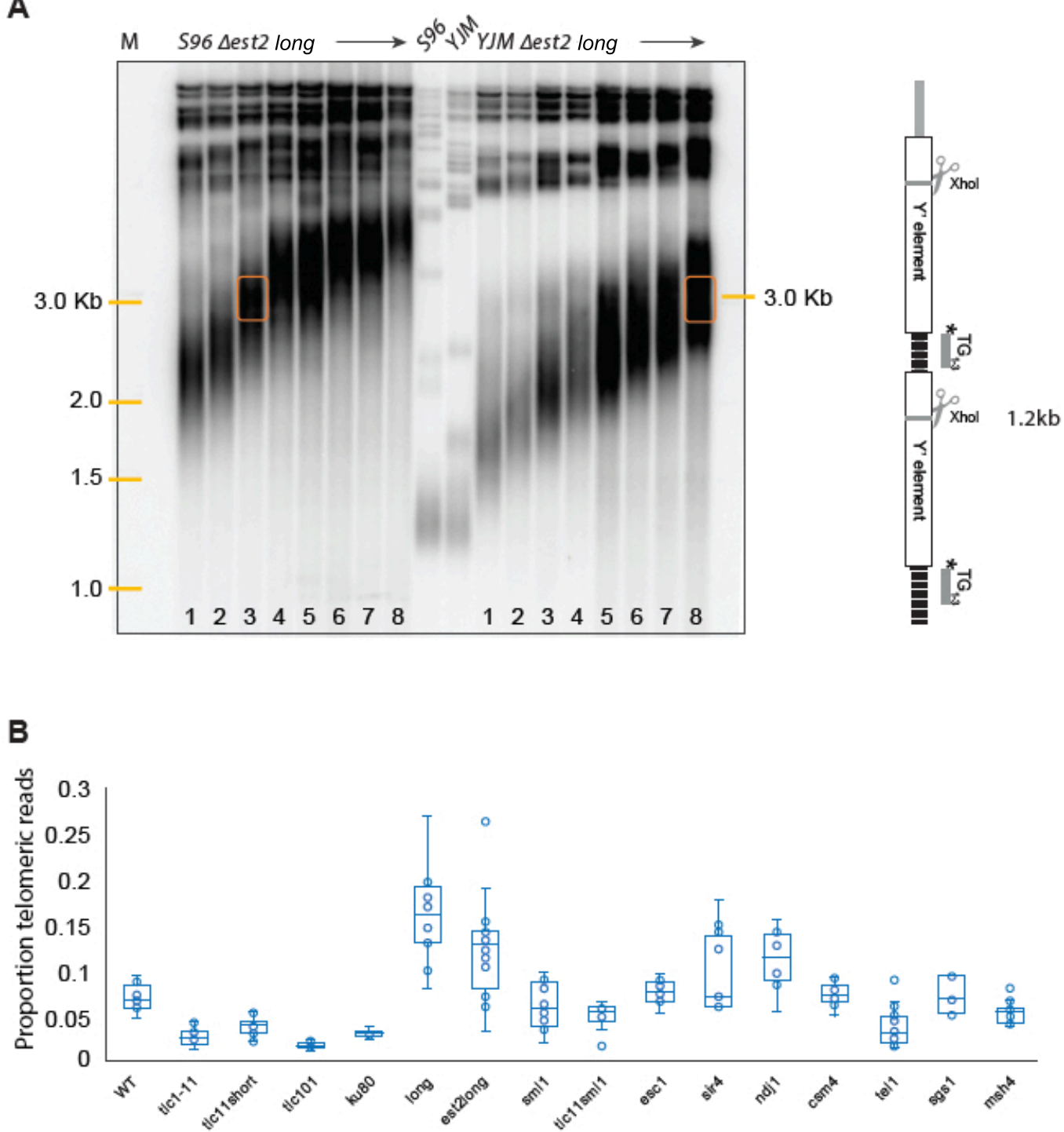

Figure $\mathrm{S} 1$ related to Figure 2

Figure S1. (Relates to Figure 2) Construction of strains with long telomeres but no telomerase.

A) EST2 was deleted in S96 and YJM789 parents and plasmid-borne Cdc13-Est2 fusion protein was integrated at $C D C 13$. Cells were serially passed 8 times (totaling about 160 generations) to ensure extensive lengthening of telomeres. The Cdc13-Est2 fusion protein was selected against, ensuring complete loss of telomerase in a long-telomere context. S96 MATa and YJM789 MAT $\alpha$ parents with similarly long telomeres were selected for mating and subsequent sequencing of meiotic products. Shown is Southern blot analysis of terminal Xhol restriction fragments. DNA was probed with an $\gamma^{32}$ P-labeled 5'-(TGTGGG) 4 -3' $Y^{\prime}$-specific probe. The lowest species represents the DNA fragment containing the terminal telomeric repeats. The selected parents have telomeres that are about 1750 bps, or 5x WT. B) Swarm plot of data shown in Figure 2D. 


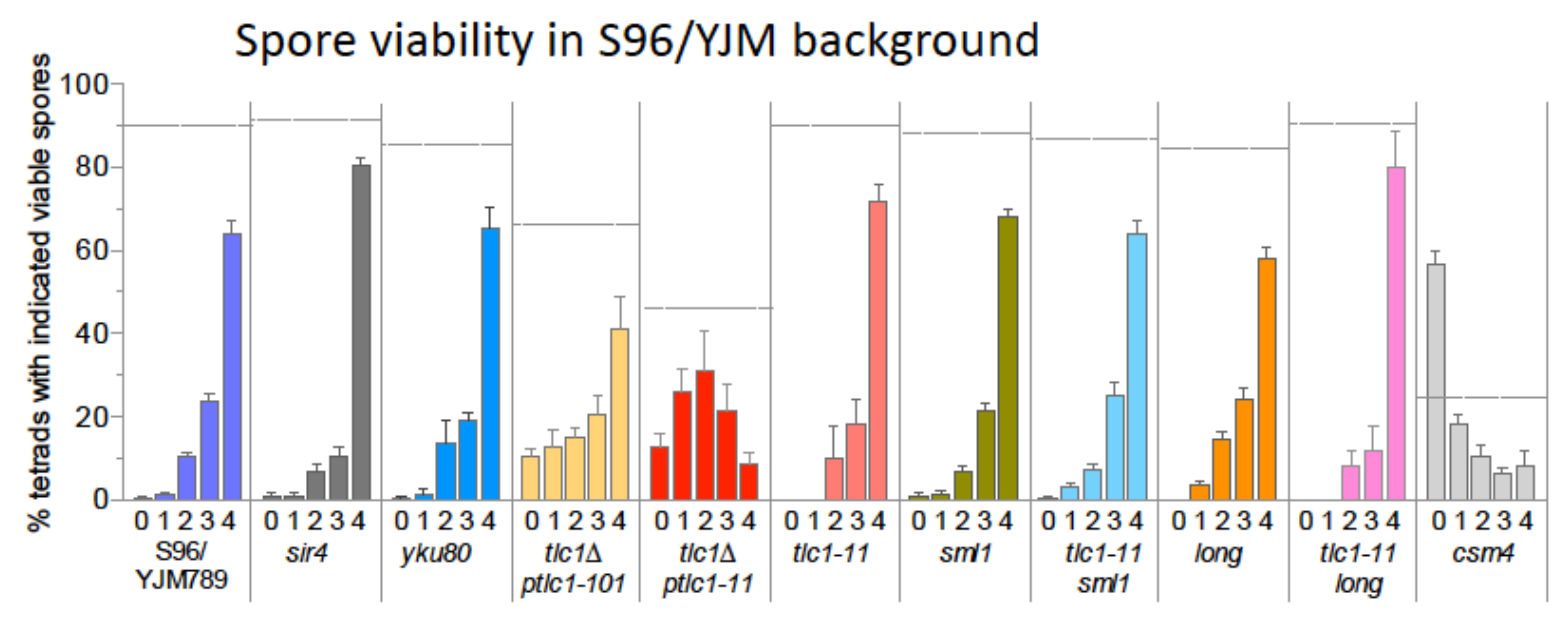

Figure S2. (Relates to Figure 3) Spore viability in hybrid strain background.

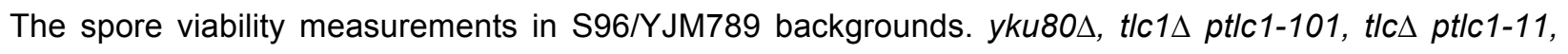
$s m / 1 \Delta$, $t / c 1-11 s m / 1 \Delta$, long, $t / c 1 \Delta$ long, and $c s m 4 \Delta$ mutants lead to spore inviability, whereas sir4 $\Delta$ does not. The $\mathrm{x}$ axis indicates $\%$ asci with $0,1,2,3$ or 4 viable spores. Horizontal dotted lines indicate total spore viability for each strain. (See Table $\mathrm{S} 1$ for more mutant spore viability data) 
A
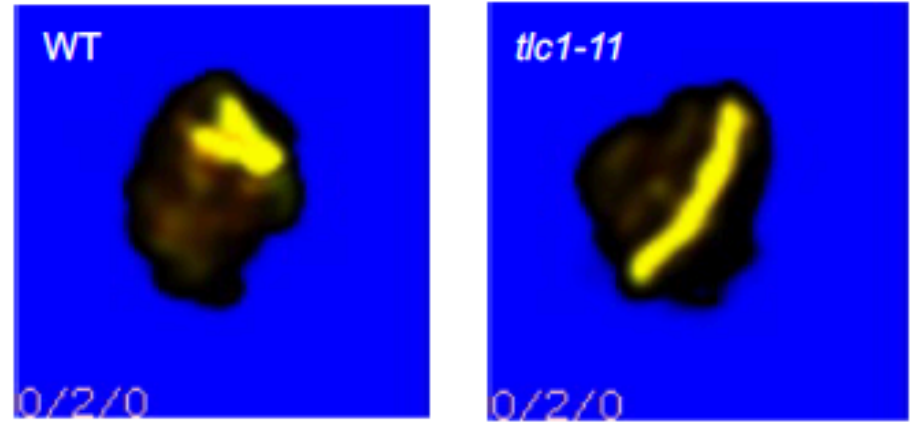

B
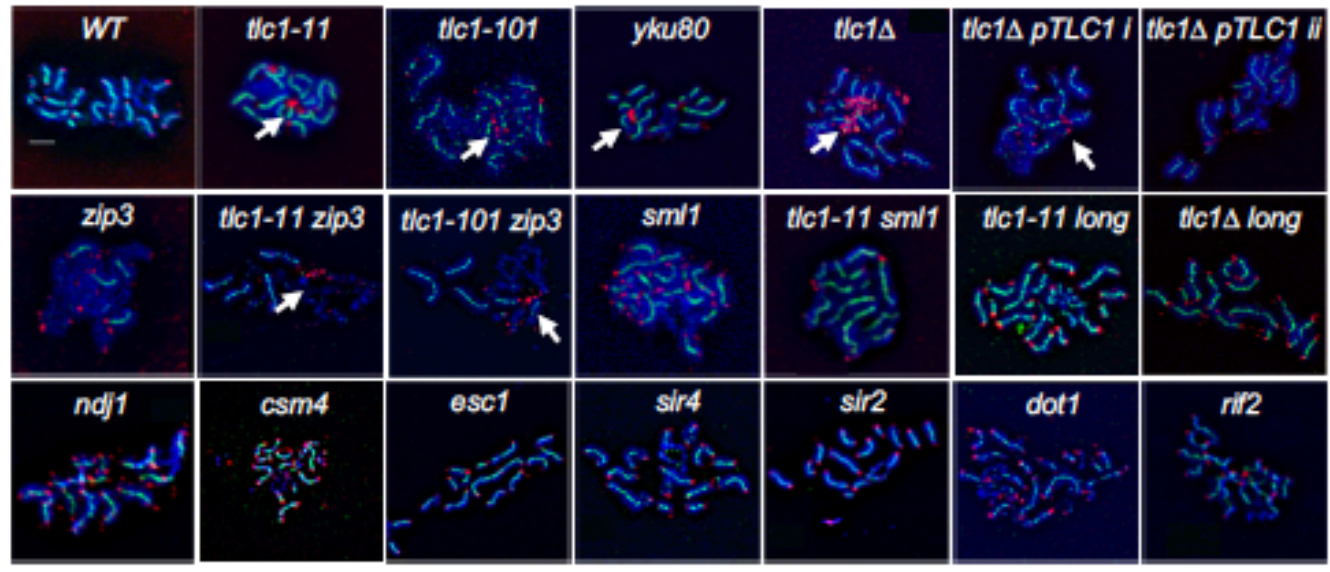

$\mathrm{C}$
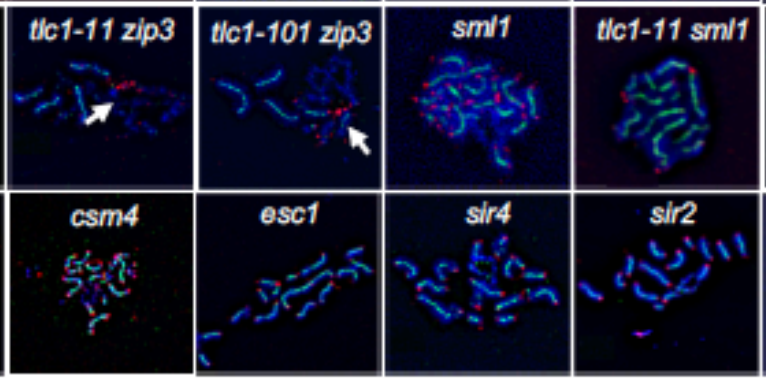

fraction nuclei with aggregated telomeres

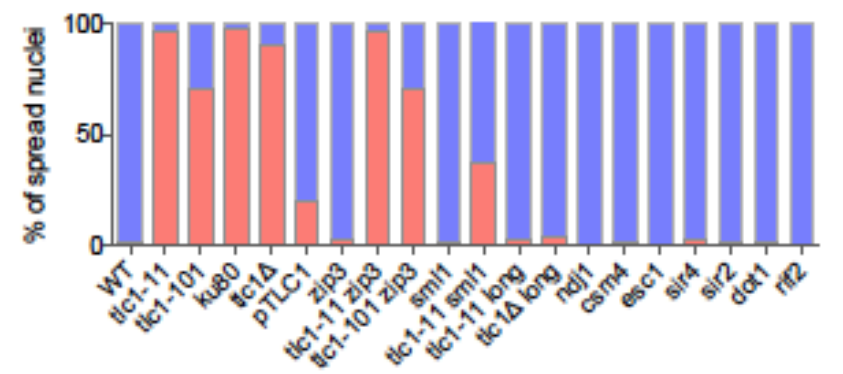

normal Rap1

Rap1 aggregates

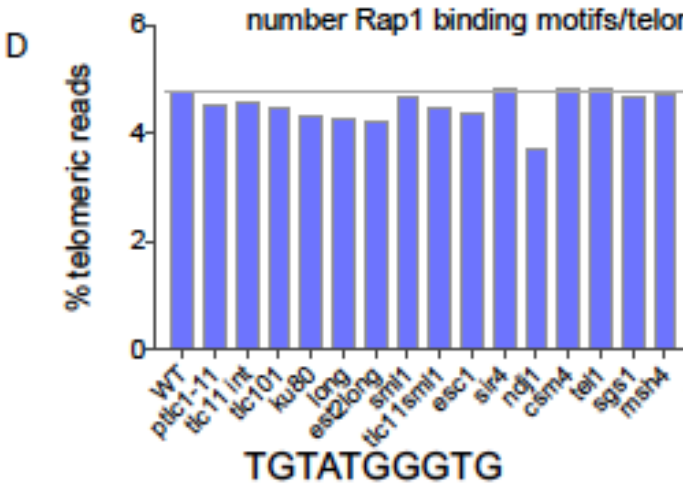

AATGTATGGATGTA

Figure S3, related to Figure 4 
Figure S3. (Relates to Figure 4) Meiotic SC movement and mutations affecting telomere length result in Rap1 aggregation.

A) Meiotic SC movement. Max intensity projection of a zip3 $\Delta$ nucleus with a single synapsed synapsed chromosome marked with Zip1-GFP for WT (right) and t/c1-11. See Supplemental Movies pair_pulls.mp4; pair_nopulls.mp4. B) Pachytene chromosomes were spread and immunostained. Blue is DAPI staining of DNA, Green shows immunostaining of Zip1-GFP and Red represents immunostaining of Rap1. White arrows indicate representative telomeric aggregates of Rap1. Rap1 binds site-specifically to doublestranded DNA throughout the genome, at discrete promoter sites (Huet et al., 1985). The highest concentration of Rap1 binding sites occurs in telomeric and sub-telomeric regions, although Rap1 is not thought to anchor telomeres to the nuclear envelope. In WT pachytene nuclei, Rap1 appears as discrete spots at the end of each chromosome (Figure $4 \mathrm{H}$ ). In t/c1-11, Rap1 staining at telomeres is faint, consistent with the shorter telomere length in the mutant. Interestingly, in most $t / c 1-11$ nuclei the Rap1 protein largely appears in large aggregates (96\%, Figure 4H). Rap1 aggregation occurs in t/c1-101 (70\%) and yku80 $(97 \%)$, but is very rare in WT $(2 \%)$ (Figure $4 \mathrm{H})$. Virtually no Rap1 aggregation is observed in deletion mutants, Sir4 or Esc1, Csm4, Ndj1, or in mutants of the sub-telomere maintenance proteins Sir2, Dot1 and the telomere length regulation protein Rif2. C) Quantification of meiotic Rap1 aggregates. Percentage of pachytene spread nuclei with RAP1 aggregates present indicated in red and fraction without aggregates are indicated in blue. D) Analysis for changes in the Rap1 binding motif in mutant strains Two Rap1 binding motifs whose sequence is shown below the graph were used to determine the percentage of reads containing these motifs in WT and mutants. Motif 1 is from the JASPAR database of transcription factor binding sites and Motif 2 is from Lascaris et al. (1999) that analyzed Rap1 binding.

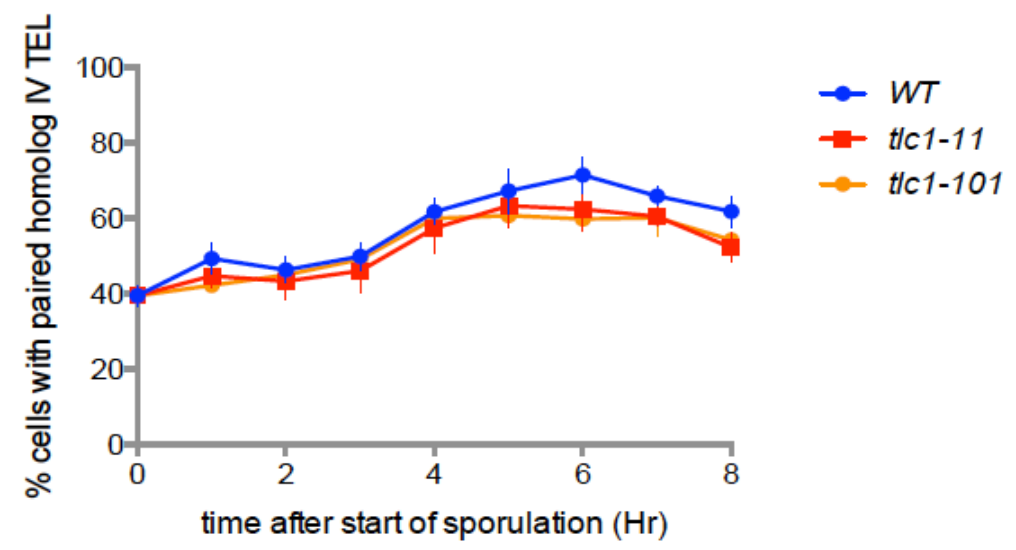

Figure S4. (Relates to Figure 5) Pairing of homologs, marked at telomeres.

Pairing of homolog IV TEL for WT, t/c1-11 and t/c1-101. Cells were gathered and fixed every hour after start of meiosis in cells with LacO repeats inserted near the telomere of each homolog. LacO foci detected with Lacl-GFP are considered paired (1 spot observed) or unpaired (two GFP-lacl spots observed) $n=1000$ per time point. 
A

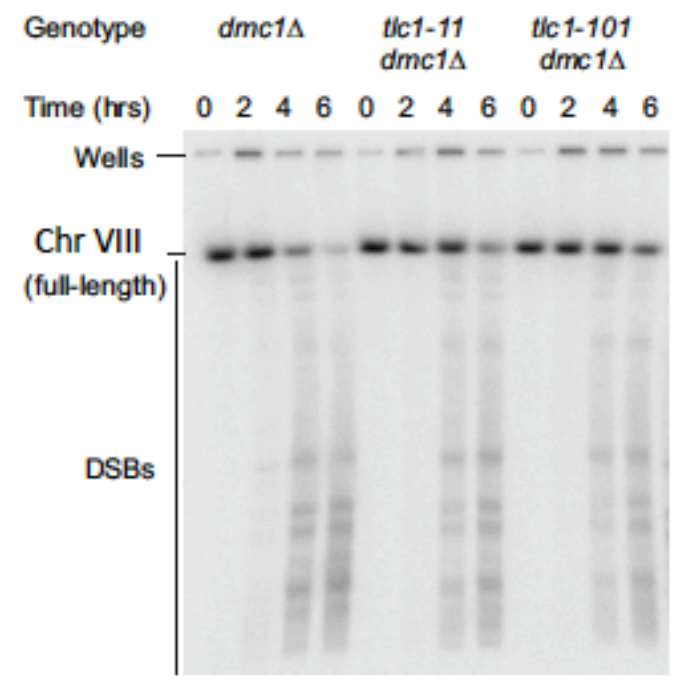

Probe: $C B P 2$
B

\section{DSBs, dmc1 whole Chr VIII}

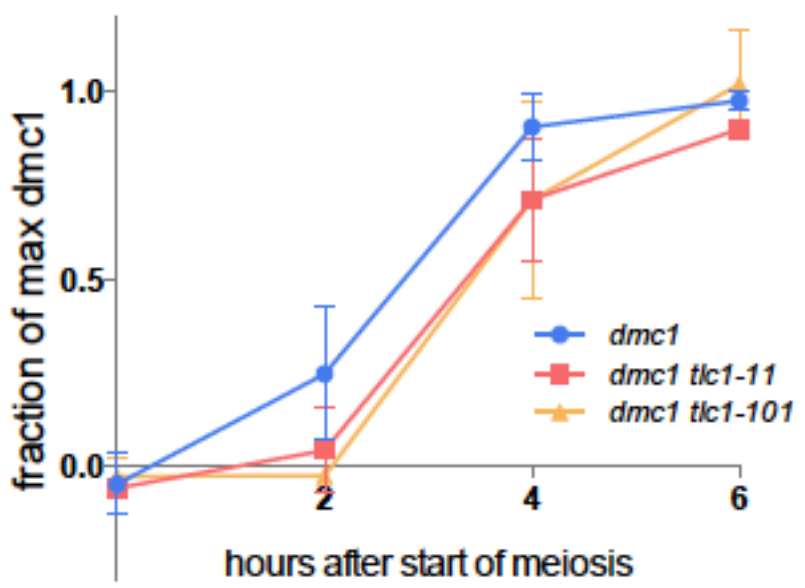

C

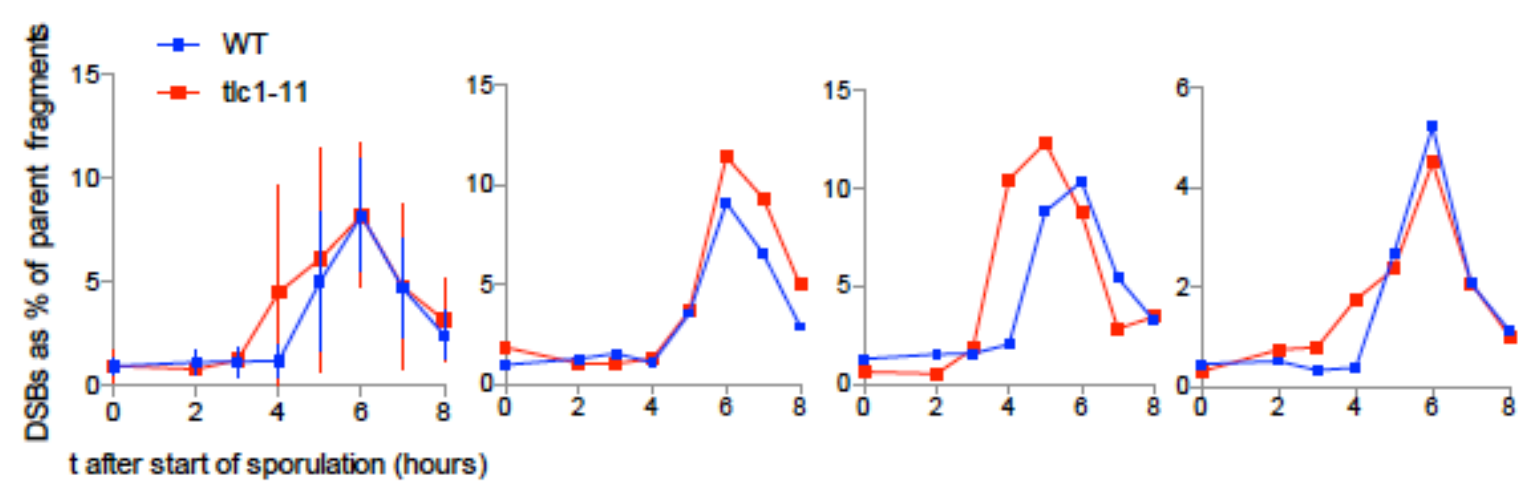

D

TLC1

tlc1-11
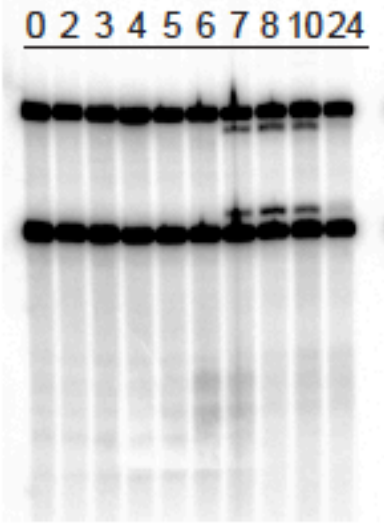

023456781024 hours
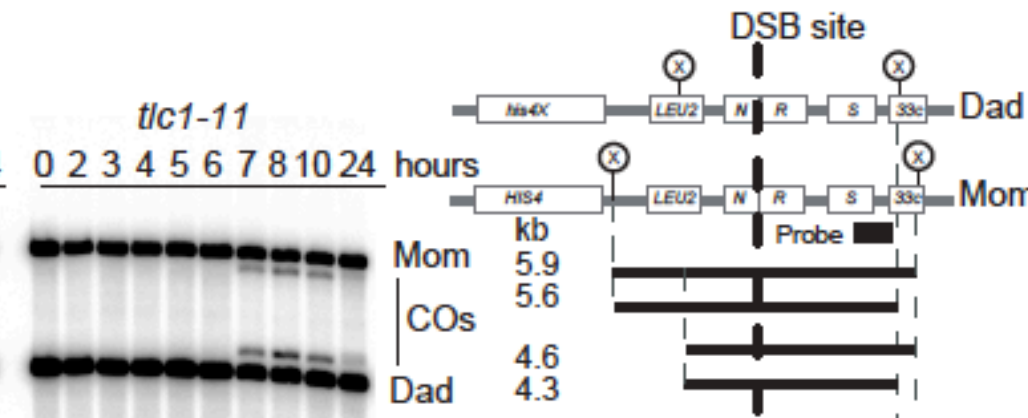

|DSBs $\begin{aligned} & 3.3 \\ & 3.0\end{aligned}$

S5: related to Figure 6

Figure S5. (Relates to Fig 6) Meiotic DSBs, on chromosome 8 and at HIS4-LEU2 hotspot. 


\begin{abstract}
A) Southern blot comparing DSB number, over time, in dmc1, t/c1-11 dmc1, t/c1-101 dmc1. A dmc1s background was used to limit DSB processing. Pulse field gel electrophoresis of DNA from samples collected every 2 hours was probed with CBP2. B) The timing and number of DSBs on Chromosome VIII in t/c1 dmc1 mutants is expressed as the fraction of $d m c 1$ maximum and is not significantly different from WT. C) Replicate time courses, showing quantification of DSBs at HIS4-LEU2 break site of Chromosome III. An average of three time courses is shown in left-most graph, with graphs of each of the three individual time courses shown to the right.

D) One-dimensional gel analysis of a blot from time course 1 (TC1) depicting meiotic recombination at the HIS4::LEU2/his4-X::LEU2-URA3 hotspot in TLC1 and t/c1-11 strains. Time course samples were treated with psoralen. DNA isolated at various timepoints was digested with Xhol and separated on a $6 \%$ agarose gel. Southern blots were hybridized with probe A ( $3^{\prime}$ end of STE50 gene open reading frame). Schematic identification of break fragments is as described by Oh et al. (2009).
\end{abstract}

Figure S6. (Relates to Figure 7) Simulation movies depicting: (Top) Homolog pairing with normal pulls (pair_pulls.mp4) and (Bottom) Homolog pairing without pulling (pair_nopulls.mp4). 